\title{
Vibration Analysis of a Piecewise-Smooth System with Negative Stiffness under Delayed Feedback Control
}

\author{
Dongmei Huang, ${ }^{1}$ Wei Li, ${ }^{1}$ Guidong Yang, ${ }^{1}$ Meijuan He, ${ }^{1}$ and Hong Dang ${ }^{2}$ \\ ${ }^{1}$ School of Mathematics and Statistics, Xidian University, Xi'an, Shaanxi 710071, China \\ ${ }^{2}$ School of Mathematics, Changzhi University, Changzhi, Shanxi 046011, China \\ Correspondence should be addressed to Dongmei Huang; dongmeihuang1@hotmail.com
}

Received 11 April 2017; Accepted 13 July 2017; Published 29 August 2017

Academic Editor: Gabriele Cazzulani

Copyright (c) 2017 Dongmei Huang et al. This is an open access article distributed under the Creative Commons Attribution License, which permits unrestricted use, distribution, and reproduction in any medium, provided the original work is properly cited.

\begin{abstract}
The principal resonance of a delayed piecewise-smooth (DPWS) system with negative stiffness under narrow-band random excitation is investigated in aspects of multiscale analysis, design methodology of the controller, and response properties. The amplitude-frequency response and steady-state moments together with the corresponding stability conditions of the controlled stochastic system are derived, in which the degradation case is also under consideration. Then, from the perspective of the equivalent damping, the comparisons of the response characteristics of the controlled system to the uncontrolled system, such as the phenomenon of frequency island, are fulfilled. Furthermore, sensitivity of the system response to feedback gain and time delay is studied and interesting dynamic properties are found. Meanwhile, the classification of the steady-state solution is also discussed. To control the maximum amplitude, the feedback parameters are determined by the frequency response together with stability boundaries which must be utilized to exclude the combinations of the unstable parameters. For the case with small noise intensity, mean-square responses present the similar characteristics to what is discussed in the deterministic case.
\end{abstract}

\section{Introduction}

The past decades have witnessed greatly increased activities and extensive developments in the theoretical and experimental research of the nonsmooth systems which are usually intractable to study due to the existence of nonsmooth factors. As a significant type of nonsmooth systems, the piecewise-smooth (PWS) system is extremely universal in the engineering field or in the application aspects with respect to vibration control [1-5]. Actually, the piecewise linearity is originally induced by the piecewise linear components included in the engineering or mechanical equipment, such as switching circuits [6], walking machines [7], and gears [8]. These components usually contain dead zone, hysteresis, saturation, and so forth. On the other hand, the inherent randomness of the mechanical systems themselves as well zas the operating environment may significantly influence the dynamic characteristics of the systems [9]. Thus, the investigation of dynamical behaviors and control problems of the PWS systems to take into both the discontinuity and the involved excitation and parameter uncertainty is of paramount importance.

Abundant research results have been achieved with respect to analysis and controller design of such PWS system [10-16]. For instance, the stability and performance of a piecewise linear system were discussed by means of the quadratic and piecewise-quadratic Lyapunov functions which could cast as convex optimization problems and linear matrix inequalities (LMI) by Hassibi and Boyd [10]. Specifically, for the discrete time piecewise affine systems, $H_{\infty}$ analysis and $H_{\infty}$ controller design for the piecewise linear systems based on a piecewise Lyapunov function were taken into account in $[11,12]$. On the other hand, it is also found that the piecewisesmooth approximation is also a powerful tool to approximate the strong nonlinear system, such as the vibration isolation system $[13,14]$. For example, a nonlinear system with negative stiffness was idealized by using the piecewise linear model in [15], in which numerical and analytical studies were discussed. The passive isolator, which is abstracted from the solid and liquid mixture (SALiM) vibration isolator, 
was approximated by a piecewise bilinear vibration isolation system in [13]. In this work, the dynamic properties and isolation performance of this system under the time-delayed velocity feedback control were explored. After that, in order to combine the cubic displacement feedback with time delay to improve the isolation performance of a high-static-lowdynamic stiffness (HSLDS) which is described by a piecewise nonlinear model, the dynamic behaviors of this system have been studied in [14]. The principal resonance of a stochastically driven elastic impact oscillator under delayed feedback control, which is described by a piecewise-smooth model, was analyzed in [16], where a criterion was put forward to avoid the appearance of jump phenomenon. As can be seen, the active control of the dynamic systems with time delay is of great concern.

In fact, time delay can be caused by finite signal transmission speeds and memory effects [17-23], the physical properties of the equipment used for control and transport delay, and filtering and processing data [21]. Time-delayed feedback control is a powerful tool for achieving a wide range of operating regimes and enhancing amplitude-frequency characteristics $[13,14,16]$; the utilization of time delay has developed for many years in vibration engineering fields. A famous example is presented by $\mathrm{Hu}$ and his coworkers [17]. They considered the primary resonance and subharmonic resonance of a forced Duffing oscillator with time delay feedback and from the perspective of vibration control. Then, a time delay position feedback controller to reduce the sway of container cranes was put forward in [23]. Recently, the abundant dynamic properties which exist in a vibration isolation system under delayed feedback control were discussed in detail in [22]. Nevertheless, the stochastic dynamics of the piecewise-smooth structure with negative stiffness under delayed feedback control, which is prone unstable in the dynamic excitations, have not been reported in the literature.

Therefore, in this paper, motivated by the work which had been done by Zou and Nagarajaiah [15], a stochastically driven PWS dynamic system with negative stiffness under nonlinear delayed feedback control will be taken into account, which can provide a comprehensive and thorough understanding of the controlled system performance. Initially, negative stiffness in applications other than earthquake has been proposed and used at small scale for the vibration isolation of equipment by Molyneux [24]. With the purpose of applying the negative stiffness concept in the massive structures, like bridges and buildings, weakening the demand of preload force and packaging the negative stiffness device in a system are necessary, and for satisfying these requirements the negative stiffness device (NSD) was developed [25]. The NSD consists of a self-contained highly compressed spring in a magnification mechanism with double negative stiffness, and a mechanism with gap spring assembly (GSA) which delays the engagement of negative stiffness until the system undergoes a specified displacement [25-28].

Without loss of generality, the principle of a NSD with an active vibration controller is presented as a mechanical model in Figure 1. As can be seen, it contains a precompressed spring with stiffness $K$, and whether the mass moves left or right, the precompressed spring will further push it in the same

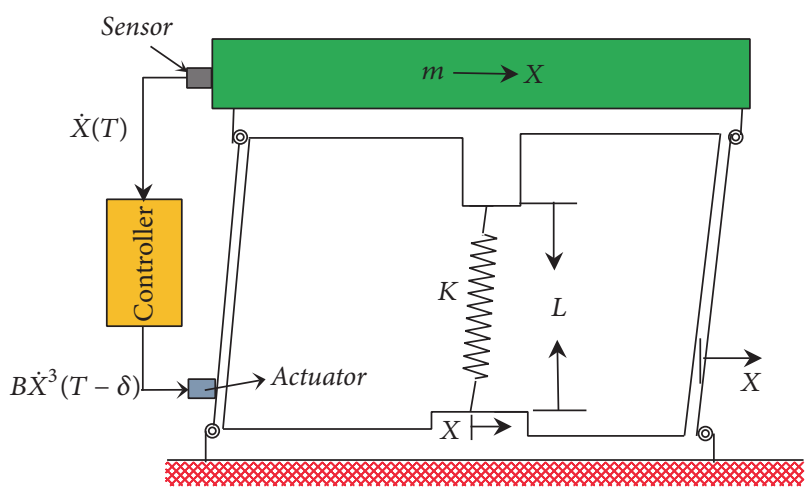

FIgURE 1: The mechanical model with negative stiffness device (NSD) under feedback control.

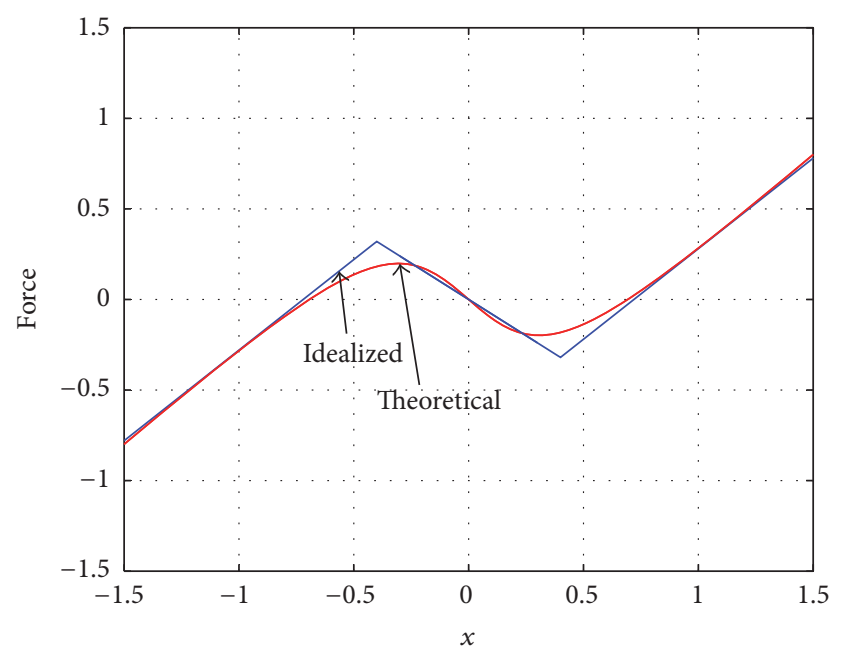

FIgURE 2: Comparison of the theoretical force and idealized force for $K=0.5, \Delta=1.1, L=0.7, K_{1}=-0.65, K_{2}=0.3$, and $d=0.55$.

direction as the displacement; this results in an interesting negative stiffness behavior [25-28].

According to the work of Zou and Nagarajaiah [15], the restoring force is assumed in the following form:

$$
h_{T}(X)=K\left(1-\frac{L+\Delta}{\sqrt{L^{2}+X^{2}}}\right) X \text {, }
$$

where $\Delta$ is the precompressed length of the spring in Figure 1. And based on [15], the dynamic system with negative stiffness is idealized as a piecewise linear system in the present paper and the comparison of the idealized force-displacement with the theoretical force-displacement loop is shown in Figure 2.

The spring force should be

$$
\begin{aligned}
& h_{I}(X) \\
& \quad=K_{1} X \\
& \quad+\Delta K[(X-d) H(X-d)+(X+d) H(-X-d)]
\end{aligned}
$$




$$
= \begin{cases}K_{2} X+\left(K_{2}-K_{1}\right) d, & X<-d, \\ K_{1} X, & -d \leq X \leq d, \\ K_{2} X-\left(K_{2}-K_{1}\right) d, & X>d,\end{cases}
$$

where $K_{1}<0, K_{2}=K_{1}+\Delta K>0, H(\cdot)$ is the Heaviside step function, and $d>0$.

Therefore, the aim of this paper is to investigate the dynamical properties of a DPWS system with negative stiffness under narrow-band random excitation. This paper is organized as follows: first, the method of multiple scales (MMS) and the moment method are used to obtain the amplitude-frequency response and the steady-state moments; stability conditions are also given to determine the selection of the stable parameters. Then, the response properties are studied by combining the stability analysis and the sensitivity of the response to the feedback parameters which are also considered. Lastly, the influence of system parameters on stochastic vibration transmissibility is illustrated.

\section{Dynamic Equation of the NSD Model and Multiscale Analysis}

According to the approximation in (2), the following dimensional single-degree-of-freedom vibration system with negative stiffness under delayed feedback control is given as follows:

$$
\begin{aligned}
m \frac{d^{2} X}{d T^{2}}+C \frac{d X}{d T}+K_{1} X \\
+\Delta K[(X-d) H(X-d)+(X+d) H(-X-d)] \\
+B\left[\frac{d X}{d T}(T-\delta)\right]^{3}=F \eta(T)
\end{aligned}
$$

where $m$ is the mass of motion object, $C$ is the damping coefficient, and $\eta(T)$ denotes the stochastic process, which is widely adopted in the engineering field [29]. $B$ and $\delta$ represent the feedback gain and the design time delay of the controller, respectively. By introducing the following nondimensional parameters and variables,

$$
\begin{aligned}
& X=x d, \\
& T=t \sqrt{\frac{M}{K_{2}}}, \\
& \delta=\tau \sqrt{\frac{M}{K_{2}},} \\
& c=\sqrt{\frac{C}{\left(M K_{2}\right)},}
\end{aligned}
$$

$$
\begin{gathered}
k_{1}=\frac{K_{1}}{K_{2}}<0, \\
\Delta k=1-\frac{K_{1}}{K_{2}}, \\
b=\frac{B}{\left(M K_{2}\right)}, \\
f=\frac{F}{\left(K_{2} d\right)} .
\end{gathered}
$$

After substituting them into (3), the following nondimensional nonlinear equation of motion is derived:

$$
\begin{aligned}
\ddot{x}+c \dot{x} & +k_{1} x \\
& +\Delta k[(x-1) H(x-1)+(x+1) H(-x-1)] \\
& +b \dot{x}^{3}(t-\tau)=f \eta(t),
\end{aligned}
$$

where dot denotes derivative with respect to nondimensional time $t$. And

$$
\eta(t)=\cos (\Omega t+\gamma W(t))
$$

where $W(t)$ is the standard Wiener process with the intensity $\gamma$. The corresponding spectrum density of $\eta(t)$ is

$$
S_{\eta}(\omega)=\frac{1}{2} \frac{\gamma^{2}\left(\Omega^{2}+\omega^{2}+\gamma^{4} / 4\right)}{\left(\Omega^{2}-\omega^{2}+\gamma^{4} / 4\right)^{2}+\omega^{2} \gamma^{4}} .
$$

When the bandwidth $\gamma$ goes to zero, the fluctuation spectrum $S_{\eta}(\omega)$ is vanishing in the entire frequency range except at the singular frequency $\omega= \pm \Omega$, where $S_{\eta}( \pm \Omega)$ goes to infinity, that is, a typical spectrum of the narrow-band random noise. In this paper, only case $\gamma$ is smaller under consideration.

The MMS [30] will be adopted to investigate the responses and its stability of system (5). Small parameters for the MMS have to be chosen based on the parameters and resonance regions of the DPWS system; hence, define $b=\varepsilon^{2} \widehat{b}, c=$ $\varepsilon^{2} \widehat{c}$, and $f=\varepsilon^{2} \widehat{f}$. Due to the negative stiffness $\left(k_{1}<0\right)$, introducing the new variable $k_{1}=1-\varepsilon^{2} \widehat{k}$, thus, (5) can be rewritten as

$$
\begin{aligned}
\ddot{x}+ & x=\varepsilon^{2}(\widehat{k} x-\widehat{c} \dot{x} \\
-\widehat{k} & {[(x-1) H(x-1)+(x+1) H(-x-1)] } \\
& \left.-\widehat{b} \dot{x}^{3}(t-\tau)+\widehat{f} \eta(t)\right) .
\end{aligned}
$$

Then, the uniformly approximate solution of (8) is presented into power series by different time scales which are distinguished by the small parameter $\varepsilon$ :

$$
x(t, \varepsilon)=\sum_{j=0}^{2} \varepsilon^{j} x_{j}\left(T_{0}, T_{1}, T_{2}\right)+O\left(\varepsilon^{3}\right),
$$


where $T_{0}=t$ is a fast time scale; $T_{1}=\varepsilon t$ and $T_{2}=\varepsilon^{2} t$ are the slow time scales. Then, substituting (9) into (8) and collecting the same powers of $\varepsilon$, the following set of equations can be formed:

$$
\begin{aligned}
\varepsilon^{0}: & D_{0}^{2} x_{0}\left(T_{0}, T_{1}, T_{2}\right)+x_{0}\left(T_{0}, T_{1}, T_{2}\right)=0, \\
\varepsilon^{1}: & D_{0}^{2} x_{1}\left(T_{0}, T_{1}, T_{2}\right)+x_{1}\left(T_{0}, T_{1}, T_{2}\right) \\
= & -2 D_{0} D_{1} x_{0}\left(T_{0}, T_{1}, T_{2}\right), \\
\varepsilon^{2}: & D_{0}^{2} x_{2}\left(T_{0}, T_{1}, T_{2}\right)+x_{2}\left(T_{0}, T_{1}, T_{2}\right) \\
= & -2 D_{0} D_{1} x_{1}\left(T_{0}, T_{1}, T_{2}\right)-2 D_{0} D_{2} x_{0}\left(T_{0}, T_{1}, T_{2}\right) \\
& -D_{1}^{2} x_{0}\left(T_{0}, T_{1}, T_{2}\right)+k x_{0}-\widehat{c} D_{0} x_{0} \\
& -\widehat{b}\left[D_{0} x_{0}\left(T_{0}-\tau, T_{1}, T_{2}\right)\right]^{3} \\
& -\widehat{k}\left[\left(x_{0}-1\right) H\left(x_{0}-1\right)+\left(x_{0}+1\right) H\left(-x_{0}-1\right)\right] \\
& +\widehat{f} \cos \left(\Omega T_{0}+\widehat{\gamma} W\left(T_{2}\right)\right),
\end{aligned}
$$

in which the property $\gamma W(t)=\gamma W\left(\varepsilon^{2} t\right) / \varepsilon=\hat{\gamma} W\left(T_{2}\right)$ is applied. The differential operators $D_{n}$ are defined as $D_{n}=$ $\partial / \partial T_{n}, n=0,1,2$. According to (10), the solution of first order can be written as

$$
x_{0}=A\left(T_{1}, T_{2}\right) \cos \left(T_{0}+\varphi\left(T_{1}, T_{2}\right)\right) \text {, }
$$

where $A\left(T_{1}, T_{2}\right)$ and $\varphi\left(T_{1}, T_{2}\right)$ are unknown complex functions determined by imposing the solvability conditions on next levels of approximation. Substituting (13) into (11) and eliminating the secular terms, it is easy to have $\partial A / \partial T_{1}=0$, $\partial \varphi / \partial T_{1}=0$, which means that $A$ and $\varphi$ only have relationship with the time variable $T_{2}$.

Denote excitation frequency as $\Omega=1+\varepsilon^{2} \sigma(\sigma$ is detuning parameter) and introduce the new variable $\Phi\left(T_{2}\right)=\sigma T_{2}-$ $\varphi\left(T_{2}\right)+\widehat{\gamma} W\left(T_{2}\right)$; then,

$$
\begin{gathered}
D_{0}^{2} x_{2}\left(T_{0}, T_{1}, T_{2}\right)+x_{2}\left(T_{0}, T_{1}, T_{2}\right)=2 A^{\prime} \sin \left(T_{0}+\varphi\right) \\
+2 A \sigma \cos \left(T_{0}+\varphi\right)-2 A \Phi^{\prime} \cos \left(T_{0}+\varphi\right)+\widehat{k} A \\
\cdot \cos \left(T_{0}+\varphi\right)+\widehat{c} A \sin \left(T_{0}+\varphi\right)+\frac{3}{4} \widehat{b} A^{3} \cos (\tau) \\
\cdot \sin \left(T_{0}+\varphi\right)-\frac{3}{4} \widehat{b} A^{3} \sin (\tau) \cos \left(T_{0}+\varphi\right)-\frac{1}{4} \widehat{b} A^{3} \\
\cdot \cos (3 \tau) \sin \left(3 T_{0}+3 \varphi\right)-\frac{1}{4} \widehat{b} A^{3} \sin (3 \tau) \\
\cdot \cos \left(3 T_{0}+3 \varphi\right)+\widehat{f} \cos \left(\Phi+T_{0}+\varphi\right)
\end{gathered}
$$

$$
\begin{aligned}
& -\widehat{k}\left[\left(A \cos \left(T_{0}+\varphi\right)-1\right) H\left(A \cos \left(T_{0}+\varphi\right)-1\right)\right. \\
& \left.+\left(A \cos \left(T_{0}+\varphi\right)+1\right) H\left(-A \cos \left(T_{0}+\varphi\right)-1\right)\right] .
\end{aligned}
$$

According to (13) and (14), the solution of (14) is

$$
\begin{aligned}
x_{2}\left(T_{0}, T_{2}\right)= & I_{1} \sin \left(T_{0}+\varphi\right)+I_{2} \cos \left(T_{0}+\varphi\right) \\
& +I_{3} \cos \left(\Omega T_{0}\right)+I_{4} \cos \left(3 T_{0}+3 \varphi\right) \\
& +I_{5} \sin \left(3 T_{0}+3 \varphi\right),
\end{aligned}
$$

where $I_{1}=I_{2}=0, I_{3}=\widehat{f} /\left(1-\Omega^{2}\right), I_{4}=\left(\widehat{b} A^{3}\left(T_{2}\right) / 32\right) \cos (3 \tau)$ and $I_{5}=\left(\widehat{b} A^{3}\left(T_{2}\right) / 32\right) \sin (3 \tau)$.

Then, the Fourier coefficients of basic harmonic terms of $\left(x_{0}-1\right) H\left(x_{0}-1\right)+\left(x_{0}+1\right) H\left(-x_{0}-1\right)$ are expressed as $G_{1}$ and $G_{2}$. The integral of $\left(x_{0}-1\right) H\left(x_{0}-1\right)+\left(x_{0}+1\right) H\left(-x_{0}-1\right)$ can be calculated in five intervals: $\left[0, \psi_{0}\right],\left[\psi_{0}, \psi_{1}\right],\left[\psi_{1}, 2 \pi-\psi_{1}\right]$, $\left[2 \pi-\psi_{1}, 2 \pi-\psi_{0}\right]$, and $\left[2 \pi-\psi_{0}, 2 \pi\right]$ based on (2), where $\psi_{0}$ and $\psi_{1}$ correspond to the phases of discontinuity points in one period $\left(A \cos \psi_{0}=1, A \cos \psi_{1}=-1\right)$. Furthermore, the coefficients can be determined by

$$
\begin{aligned}
G_{1} & =\frac{1}{\pi} \int_{0}^{2 \pi}\left[\left(x_{0}-1\right) H\left(x_{0}-1\right)\right. \\
& \left.+\left(x_{0}+1\right) H\left(-x_{0}-1\right)\right] \sin (\psi) d \psi=0, \\
G_{2} & =\frac{1}{\pi} \int_{0}^{2 \pi}\left[\left(x_{0}-1\right) H\left(x_{0}-1\right)\right. \\
& \left.+\left(x_{0}+1\right) H\left(-x_{0}-1\right)\right] \cos (\psi) d \psi=\frac{1}{\pi}[A(\pi \\
& \left.-\psi_{1}+\psi_{0}+\frac{1}{2} \sin \left(2 \psi_{0}\right)-\frac{1}{2} \sin \left(2 \psi_{1}\right)\right)-2 \\
& \left.\cdot \sin \left(\psi_{0}\right)-2 \sin \left(\psi_{1}\right)\right] .
\end{aligned}
$$

In order to eliminate the secular term in (14), the right side of (14) should not include the terms $\sin \left(T_{0}+\varphi\right)$ and $\cos \left(T_{0}+\varphi\right)$; thus it is easy to have

$$
\begin{aligned}
A^{\prime}\left(T_{2}\right)= & -\frac{\widehat{c}}{2} A\left(T_{2}\right)-\frac{3}{8} \widehat{b} A^{3}\left(T_{2}\right) \cos (\tau) \\
& +\frac{\widehat{f}}{2} \sin (\Phi), \\
A\left(T_{2}\right) \Phi^{\prime}\left(T_{2}\right)= & \sigma A\left(T_{2}\right)+\frac{1}{2} \widehat{k} A\left(T_{2}\right) \\
& -\frac{3}{8} \widehat{b} A^{3}\left(T_{2}\right) \sin (\tau) \\
& +\frac{\widehat{f}}{2} \cos (\Phi)-\frac{\widehat{k}}{2} G_{2} \\
& +\widehat{\gamma} A\left(T_{2}\right) W^{\prime}\left(T_{2}\right) .
\end{aligned}
$$


2.1. Deterministic and Stochastic Responses. For the deterministic case, the steady-state solutions of system (5) are denoted as $A_{0}$ and $\Phi_{0}$ with the conditions $A^{\prime}=0$ and $\Phi^{\prime}=0$ being satisfied:

$$
\begin{aligned}
& \frac{\widehat{c}}{2} A_{0}+\frac{3}{8} \widehat{b} A_{0}^{3} \cos (\tau)=\frac{\widehat{f}}{2} \sin \left(\Phi_{0}\right), \\
& -\sigma A_{0}-\frac{1}{2} \widehat{k} A_{0}+\frac{3}{8} \widehat{b} A_{0}^{3} \sin (\tau)+\left.\frac{\widehat{k}}{2} G_{2}\right|_{A=A_{0}} \\
& \quad=\frac{\widehat{f}}{2} \cos \left(\Phi_{0}\right) .
\end{aligned}
$$

Then, it is straightforward to obtain the amplitudefrequency equation by the application of the formula $\sin ^{2} \Phi_{0}+\cos ^{2} \Phi_{0}=1$ :

$$
\begin{aligned}
& {\left[\frac{\widehat{c}}{2} A_{0}+\frac{3}{8} \widehat{b} A_{0}^{3} \cos (\tau)\right]^{2}} \\
& \quad+\left[-\sigma A_{0}-\frac{1}{2} \widehat{k} A_{0}+\frac{3}{8} \widehat{b} A_{0}^{3} \sin (\tau)+\left.\frac{\widehat{k}}{2} G_{2}\right|_{A=A_{0}}\right]^{2} \\
& \quad=\frac{\widehat{f}^{2}}{4} .
\end{aligned}
$$

Herein, in order to discuss the effect of the narrowband noise $(\hat{\gamma} \neq 0)$ on the DPWS system, introduce the perturbation terms $A_{1}$ and $\Phi_{1}$ :

$$
\begin{aligned}
& A=A_{0}+A_{1}, \\
& \Phi=\Phi_{0}+\Phi_{1},
\end{aligned}
$$

where $A_{0}$ and $\Phi_{0}$ are governed by (18). Substituting (20) into (17a) and (17b) and neglecting the nonlinear terms, the linearization stochastic differential equations of (17a) and (17b) at $A_{0}$ and $\Phi_{0}$ can be obtained:

$$
\begin{aligned}
d A_{1} & =\left[-\frac{\widehat{c}}{2}-\frac{9}{8} \widehat{b} A_{0}^{2} \cos (\tau)\right] A_{1} d T_{2}+\frac{\widehat{f}}{2} \cos \left(\Phi_{0}\right) \\
\cdot & \Phi_{1} d T_{2}, \\
d \Phi_{1} & \\
= & {\left[\frac{\sigma}{A_{0}}+\frac{\widehat{k}}{2 A_{0}}-\frac{9}{8} \widehat{b} A_{0} \sin (\tau)-\left.\frac{\widehat{k}}{2 A_{0}} \frac{d G_{2}}{d A}\right|_{A=A_{0}}\right] } \\
\cdot & A_{1} d T_{2}-\frac{\widehat{f}}{2 A_{0}} \sin \left(\Phi_{0}\right) \Phi_{1} d T_{2}+\widehat{\gamma} d W\left(T_{2}\right),
\end{aligned}
$$

where $d G_{2} / d A=(1 / \pi)\left\{\pi-\psi_{1}+\psi_{0}+(1 / 2)\left[\sin \left(2 \psi_{0}\right)-\right.\right.$ $\left.\sin \left(2 \psi_{1}\right)\right]+A\left[d \psi_{0} / d A-d \psi_{1} / d A+\cos \left(2 \psi_{0}\right)\left(d \psi_{0} / d A\right)-\right.$ $\left.\cos \left(2 \psi_{1}\right)\left(d \psi_{1} / d A\right)\right]-2 \cos \left(\psi_{0}\right)\left(d \psi_{0} / d A\right)-2 \cos \left(\psi_{1}\right)\left(d \psi_{1} /\right.$ $d A)\}$.

Furthermore, the method of moment is applied to consider the first-order and second-order steady-state moments of the stochastic system, and the following property is also utilized:

$$
\begin{aligned}
\frac{d E A_{1}}{d T_{2}} & =\frac{d E \Phi_{1}}{d T_{2}}=\frac{d E\left(A_{1}^{2}\right)}{d T_{2}}=\frac{d E\left(\Phi_{1}^{2}\right)}{d T_{2}} \\
& =\frac{d E\left(A_{1} \Phi_{1}\right)}{d T_{2}}=0,
\end{aligned}
$$

where $E(\cdot)$ denotes the mathematical expectation. Then, the first-order steady-state moments of $A_{1}$ and $\Phi_{1}$ are $E A_{1}=$ $E \Phi_{1}=0$, and it also has

$$
\begin{aligned}
\frac{d E\left(A_{1}^{2}\right)}{d T_{2}}= & 2 C_{1} E\left(A_{1}^{2}\right)+2 C_{3} E\left(A_{1} \Phi_{1}\right)=0, \\
\frac{d E\left(\Phi_{1}^{2}\right)}{d T_{2}}= & 2 C_{2} E\left(A_{1} \Phi_{1}\right)-2 C_{4} E\left(\Phi_{1}^{2}\right)+\widehat{\gamma}^{2}=0, \\
\frac{d E\left(A_{1} \Phi_{1}\right)}{d T_{2}}= & \left(C_{1}-C_{4}\right) E\left(A_{1} \Phi_{1}\right)+C_{3} E\left(\Phi_{1}^{2}\right) \\
& +C_{2} E\left(A_{1}^{2}\right)=0,
\end{aligned}
$$

where $C_{1}=-\widehat{c} / 2-(9 / 8) \widehat{b} A_{0}^{2} \cos (\tau), C_{2}=\sigma / A_{0}+\widehat{k} / 2 A_{0}-$ $(9 / 8) \hat{b} A_{0} \sin (\tau)-\left.\left(\hat{k} / 2 A_{0}\right)\left(d G_{2} / d A\right)\right|_{A=A_{0}}, C_{3}=-\sigma A_{0}-$ $(1 / 2) \widehat{k} A_{0}+(3 / 8) \widehat{b} A_{0}^{3} \sin (\tau)+\left.(\widehat{k} / 2) G_{2}\right|_{A=A_{0}}, C_{4}=\widehat{c} / 2+$ $(3 / 8) \hat{b} A_{0}^{2} \cos (\tau)$.

Therefore, the second-order moment of $A_{1}$ is also easy to derive

$$
E\left(A_{1}^{2}\right)=\frac{\hat{\gamma}^{2} C_{3}^{2}}{2 C_{1} C_{2} C_{3}-2 C_{2} C_{3} C_{4}+2 C_{4} C_{1}^{2}-2 C_{4}^{2} C_{1}} .
$$

Furthermore, the first-order and second-order steady-state moments of amplitude $A$ are derived as follows:

$$
\begin{aligned}
& E A=E\left(A_{0}+A_{1}\right)=A_{0}, \\
& \begin{aligned}
E\left(A^{2}\right)=A_{0}^{2}+E\left(A_{1}^{2}\right) \\
\quad=A_{0}^{2}+\frac{\hat{\gamma}^{2} C_{3}^{2}}{2 C_{1} C_{2} C_{3}-2 C_{2} C_{3} C_{4}+2 C_{4} C_{1}^{2}-2 C_{4}^{2} C_{1}} .
\end{aligned}
\end{aligned}
$$

2.2. Discussion of the Stability. For the deterministic case $(\hat{\gamma}=$ 0 ), by means of the following characteristic equation of the coefficient matrix of (17a) and (17b),

$$
\lambda^{2}+\left[\frac{\widehat{c}}{2}+\frac{3}{2} \widehat{b} A_{0}^{2} \cos (\tau)\right] \lambda+N=0 .
$$

In accordance with the Routh-Hurwitz criterion [31], the steady-state solutions of (19) are asymptotically stable 
if the following two inequalities are satisfied simultaneously:

$$
\begin{aligned}
M= & \frac{\widehat{c}}{2}+\frac{3}{2} \widehat{b} A_{0}^{2} \cos (\tau)>0 \\
N= & \frac{\widehat{c}^{2}}{4}+\frac{3}{4} \widehat{c} \widehat{b} A_{0}^{2} \cos (\tau)+\frac{27}{64} \widehat{b}^{2} A_{0}^{4}+\sigma^{2}+\sigma \widehat{k} \\
& -\frac{3}{2} \sigma \widehat{b} A_{0}^{2} \sin (\tau)-\left.\frac{1}{2} \frac{\sigma \widehat{k}}{A_{0}} G_{2}\right|_{A=A_{0}}+\frac{1}{4} \widehat{k}^{2} \\
& -\frac{3}{4} \widehat{k} \widehat{b} A_{0}^{2} \sin (\tau)-\left.\frac{1}{4} \frac{\widehat{k}^{2}}{A_{0}} G_{2}\right|_{A=A_{0}} \\
& +\left.\frac{9}{16} \widehat{b} \widehat{k} A_{0} \sin (\tau) G_{2}\right|_{A=A_{0}}-\left.\frac{1}{2} \sigma \widehat{k} \frac{d G_{2}}{d A}\right|_{A=A_{0}} \\
& -\left.\frac{1}{4} \widehat{k}^{2} \frac{d G_{2}}{d A}\right|_{A=A_{0}}+\left.\frac{3}{16} \widehat{k} \widehat{b} A_{0}^{2} \sin (\tau) \frac{d G_{2}}{d A}\right|_{A=A_{0}} \\
& +\left.\frac{1}{4} \frac{\widehat{k}^{2}}{A_{0}}\left(G_{2} \frac{d G_{2}}{d A}\right)\right|_{A=A_{0}}>0 .
\end{aligned}
$$

Correspondingly, for the stochastic case $(\widehat{\gamma} \neq 0)$, the necessary condition for the existence of the second-order moments from (26) is

$$
C_{1} C_{2} C_{3}-C_{2} C_{3} C_{4}+C_{4} C_{1}^{2}-C_{4}^{2} C_{1}>0 .
$$

Then, the coefficient matrix of (23) is

$$
\left[\begin{array}{ccc}
2 C_{1} & 0 & 2 C_{3} \\
0 & -2 C_{4} & 2 C_{2} \\
C_{2} & C_{3} & C_{1}-C_{4}
\end{array}\right] .
$$

Alternatively, the characteristic equation of matrix (30) combined with the Routh-Hurwitz criterion, which is applied to identify the stability of second-order steady-state moments, is employed for the calculations required. And the corresponding characteristic equation is

$$
\begin{aligned}
\lambda^{3}- & 3\left(C_{1}-C_{4}\right) \lambda^{2} \\
& +\left[2 C_{1}^{2}+2 C_{4}^{2}-8 C_{1} C_{4}-4 C_{2} C_{3}\right] \lambda+4 C_{1}^{2} C_{4} \\
& -4 C_{1} C_{4}^{2}-4 C_{2} C_{3} C_{4}-4 C_{1} C_{2} C_{3}=0 .
\end{aligned}
$$

The nontrivial steady-state moments are asymptotically stable, when the following inequalities hold:

$$
\begin{aligned}
& C_{1}<C_{4}, \\
& -3 C_{1}^{3}+13 C_{1}^{2} C_{4}-13 C_{1} C_{4}^{2}-4 C_{2} C_{3} C_{4}+4 C_{1} C_{2} C_{3} \\
& \quad+3 C_{4}^{2}>0 .
\end{aligned}
$$

\section{Analysis of the Response Properties}

To examine the primary resonances of the DPWS system under stochastic excitation and illustrate the efficacy of the proposed procedure, the numerical simulation is performed by numerically integrating the DPWS system with negative stiffness using fourth-order Runge-Kutta algorithm. Throughout our study the basic parameters of system (5) are set as $c=0.08, b=0.02, k_{1}=-0.8$, unless other case is indicated.

3.1. Amplitude-Frequency Response and Stability Analysis. To demonstrate how the time delay influences the motion related to the complex system structure, the curves of the amplitudefrequency response for time delay $\tau=\pi / 2$ and $\tau=3 \pi / 4$ are depicted in Figure 3. Note that the curves of amplitudefrequency response below the line $A_{0}=1$ are the special case, which are derived in the degradation case; that is to say, the gap $d$ is infinite; thus, the nondimensional term $\left(x_{0}-1\right) H\left(x_{0}-\right.$ $1)+\left(x_{0}+1\right) H\left(-x_{0}-1\right)$ is always zero.

As can be seen in Figure 3(a), the case when the time delay is $\tau=\pi / 2$ is firstly considered, and the responses are continuous curves with the apparent maximum in spite of different values of excitation amplitude $f$. Particularly, when $f$ is relatively smaller, such as $f=0.08$, the response curve will be below the line $A_{0}=1$; that is to say, (19) only has the imaginary root, and this point could be verified in next subsection. As $f$ increases, the response will be triple-valued since there exists one branch which is the degradation case.

However, for the case of $\tau=3 \pi / 4$ being presented in Figure 3(b), the phenomenon of frequency island under smaller values of $f$, such as $f=0.08$, is observed, which is caused by the fact that there exist branches with multiple solutions satisfying the extreme condition $d \Omega / d A_{0}=0$. Nevertheless, as $f$ decreases, only the island branch is presented in the response curve; it is due to the fact that the main branch is below line $A_{0}=1$ as plotted for $f=0.05$. The existence of this kind of response can be further analyzed in the next subsection.

Finally, the stability and classification of the steady-state solutions are also discussed in Figure 3; since the response below line $A_{0}=1$ is the degradation case, only the region above the line $A_{0}=1$ is analyzed. When $N$ is negative, all these points are the saddle points which are always unstable; naturally, as shown in Figure 3, the numerical results could not obtain these points. Nevertheless, when $N$ is positive, the unstable solutions can also occur, which happens in the region satisfying $M>0$. The positive values of $M$ are related to the part of the plane above the dash line in Figure 3, corresponding to the zero value of $M$. Furthermore, in the stability regions, the stable foci and nodes are included, which are classified by the boundary line $M^{2}-4 N=0$. These findings are checked numerically by carrying out direct integration of (5). Steady-state numerical results obtained by integrating (5) directly are depicted by circles, confirming the response characteristics in Figure 3.

3.2. Verification of the Response in Section 3.1. As well known that if there are two points on the frequency response curve with a vertical slope, corresponding to $d \Omega / d A_{0}=0$, the existence of the jump can be observed. Based on this theory, the response characteristic in Figure 3 can be verified. Firstly, 


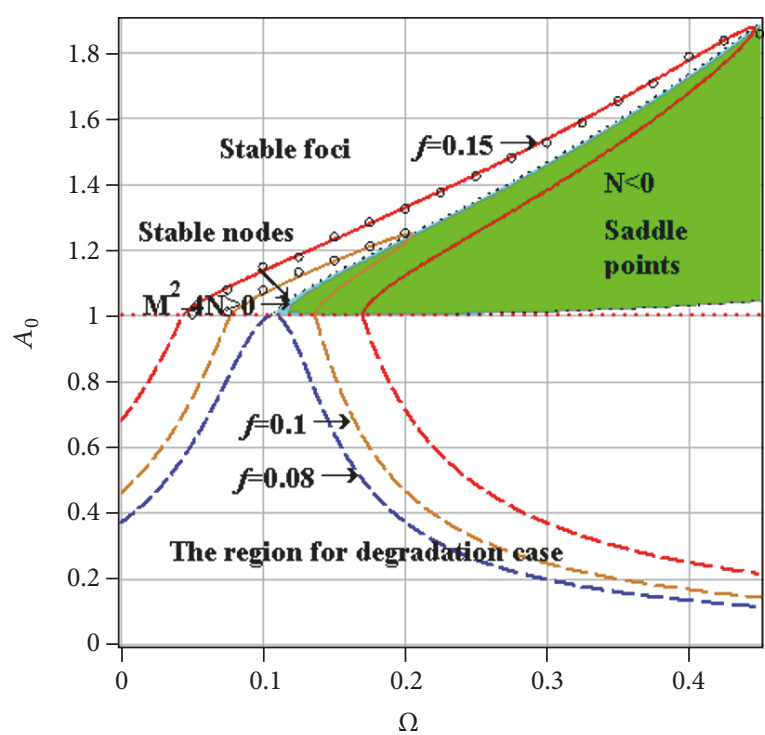

(a)

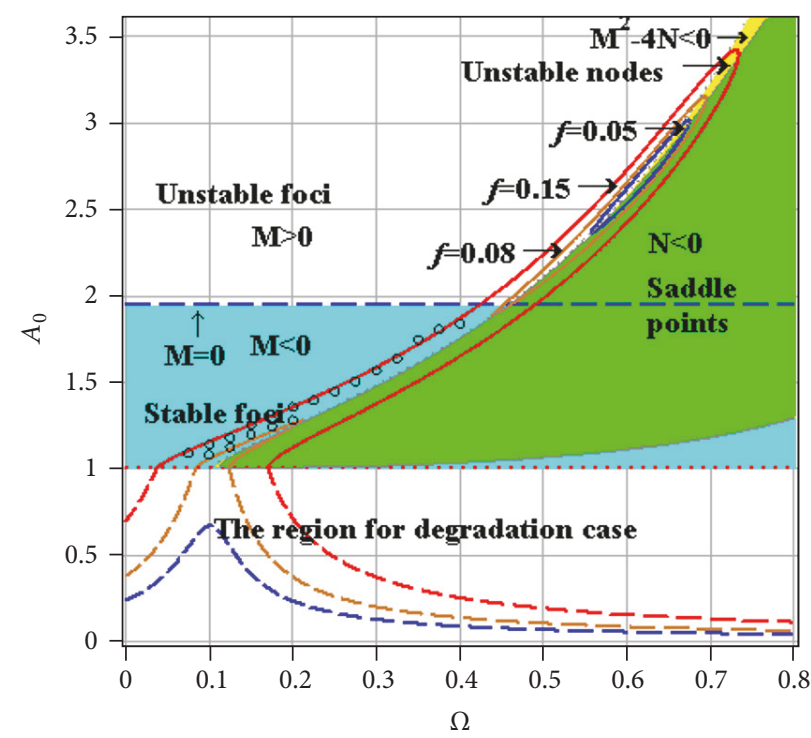

(b)

FIGURE 3: Comparison of frequency responses under different external excitation amplitudes. Solid lines: the curves of frequency responses; dash lines: the curves of degradation frequency responses. Circles present the numerical results. (a) $\tau=\pi / 2$; (b) $\tau=3 \pi / 4$.

according to (19), the excitation frequency can be derived as follows:

$$
\begin{aligned}
\Omega=1 & -\frac{1}{2} \widehat{k}+\frac{3}{8} \widehat{b} A_{0}^{2} \sin (\tau)+\frac{\left.\widehat{k} G_{2}\right|_{A=A_{0}}}{2 A_{0}} \\
& \pm \frac{\sqrt{\hat{f}^{2} / 4-\left(\widehat{c} A_{0} / 2+3 \widehat{b} A_{0}^{3} \cos (\tau) / 8\right)^{2}}}{A_{0}} .
\end{aligned}
$$

Differentiating both sides of (33) with respect to $A_{0}$ and substituting $d \Omega / d A_{0}=0$ yield

$$
\begin{aligned}
& \frac{3}{4} \widehat{b} A_{0} \sin (\tau)-\frac{\left.\widehat{k} G_{2}\right|_{A=A_{0}}}{2 A_{0}^{2}}+\left.\frac{\widehat{k}}{2 A_{0}} \frac{d G_{2}}{d A}\right|_{A=A_{0}} \\
& \pm\left[-\frac{\sqrt{\hat{f}^{2} / 4-\left(\widehat{c} A_{0} / 2+3 \widehat{b} A_{0}^{3} \cos (\tau) / 8\right)^{2}}}{A_{0}^{2}}\right. \\
& \left.-\frac{\left(\widehat{c} A_{0} / 2+3 \widehat{b} A_{0}^{3} \cos (\tau) / 8\right)\left(\widehat{c} / 2+9 \widehat{b} A_{0}^{2} \cos (\tau) / 8\right)}{\sqrt{\widehat{f}^{2} / 4-\left(\widehat{c} A_{0} / 2+3 \widehat{b} A_{0}^{3} \cos (\tau) / 8\right)^{2}} A_{0}}\right] \\
& =0 .
\end{aligned}
$$

As can be seen in Figure 3(a), when $f=0.08$ the system response is the degradation case. Actually, as shown in Figure 4(a), once $f<0.0828$, the vertical slope does not exist; thus, only the degradation case exists, and this is consistent with the result in Figure 3(a).
Corresponding to Figure 3(b), when $f$ lies in part I of Figure 4(b) (in the interval $f \in[0,0.07055]$ ), which is similar to the case of $f=0.05$, only the close-loop remains in the DPWS system; thus, there are two points on the frequency response curve with a vertical slope; when $f \in$ $[0.07055,0.08455]$, it is the response of the frequency island; therefore, there are three points on the frequency response curve with $d \Omega / d A_{0}=0$ in part II; when $f$ is greater than 0.08455 , there exists the double-valued response; hence, two points on the frequency response curve satisfy the condition $d \Omega / d A_{0}=0$.

\section{Sensitivity Analysis of the Frequency Response to Feedback Parameters}

4.1. Comparison between Frequency Responses of Controlled and Uncontrolled Systems. According to the results in [13], the equivalent damping of the system can be defined as

$$
\zeta_{\text {eq }}=\frac{c}{2}+\frac{3}{8} b A_{0}^{2} \cos (\tau) .
$$

Obviously, when $\tau \in[0, \pi / 2]$ and $[3 \pi / 2,2 \pi]$, the inequality $c / 2 \leq \zeta_{\text {eq }} \leq c / 2+3 b A_{0}^{2} \cos (\tau) / 8$ is satisfied. Note that $c / 2+$ $3 b A_{0}^{2} / 8$ is the damping without time delay. Hence, compared with uncontrolled system, the velocity feedback with nonzero time delay is capable of increasing the damping value and decreasing the response amplitude, but less efficient than the feedback without time delay. However, when $\tau \in[\pi / 2,3 \pi / 2]$, $\zeta_{\mathrm{eq}} \leq c / 2$ is satisfied; that is, the equivalent damping is smaller than the linear damping of the uncontrolled system; thus such feedback control fails to suppress the displacement response in the resonance region. 


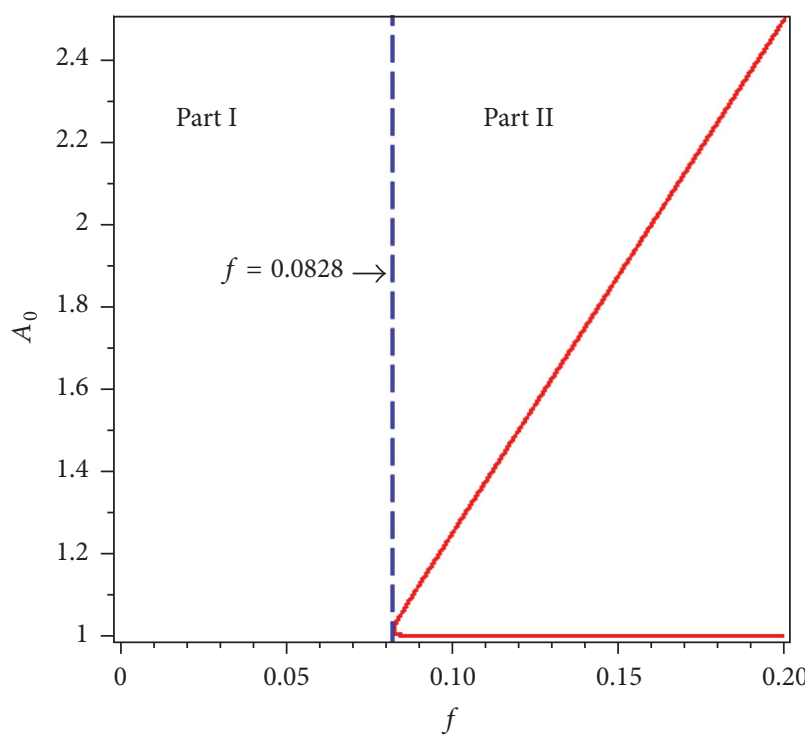

(a)

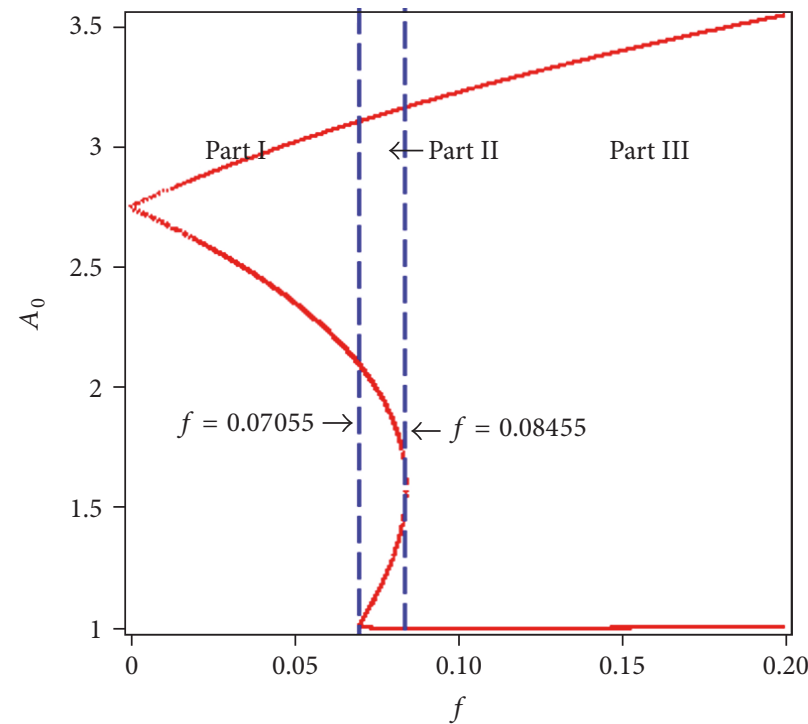

(b)

FIGURE 4: Verification of the response properties shown in Figure 3. (a) $\tau=\pi / 2$; (b) $\tau=3 \pi / 4$.

As time delay changes in the interval $[0,2 \pi]$, the variation of the response amplitude is shown in Figure 5. Meanwhile, as a comparison, the uncontrolled response $(b=0)$ is also depicted. It is consistent with the variation of equivalent damping $\zeta_{\mathrm{eq}}$. The shaded regions represent the unstable regions of the controlled system.

4.2. Effects of Time Delay and Feedback Gain on the Response. As can be discussed in Section 4.1, time delays have an extremely important effect on the system response; therefore, it is meaningful to analyze the effect of time delay and feedback gain specifically. Taken $\Omega=1.1$ as an example, here, two different cases of feedback gains are considered $(b=0.05$ and $b=0.1$ ). Several frequency response curves are plotted by the solid lines in Figure 6. The shaded regions (unstable regions I and II) present the unstable regions.

When the feedback gain is smaller $(b=0.05)$, under larger values of excitation amplitude $f$, such as $f=2.2$, the response is separated into two parts: the higher amplitude part which is a closed-loop in every period and the lower amplitude part which is one single-valued continuous curve in the whole plane. As $f$ decreases, the left and right branches of the lower amplitude part in every period become closer to each other. There is a value of amplitude $f$ when they coalesce and then separate into independent parts as plotted for $f=$ 2.0 in every period, which is similar to the independent time delay island. Nevertheless, only the closed-loop remains when $f=1.5$, which is an interesting phenomenon.

In order to compare with the results obtained in the case of $b=0.05$, the other case for $b=0.1$ is also taken into consideration. It is found that, under larger values of $f(f=$ 2.2 ), the response is one continuous curve in the whole plane rather than including two separated parts. As $f$ decreases, the response characteristic is analogous to the case of $b=0.05$.
It is also noticeable that the steady-state solutions in the unstable region II are the saddle points; the corresponding boundary points result in the appearance of the saddlenode bifurcation. The steady-state solutions inside the nodes region are associated with nodes, and outside this region these solutions are foci. The points on the boundaries of the unstable region I are Hopf bifurcation points, which result in the quasiperiodic motion in the original system. Moreover, the boundaries of unstable region I are the division lines for stable and unstable nodes and foci.

In order to deeply understand the effect of feedback gain and time delay on the closed-loop response, the continuous changes of the parameters are depicted as surface plots in Figure 7 under four different cases of excitation amplitude $f$. With the decrease of $f$, the phenomenon shown in Figure 6 can be observed more clearly and intuitively. Vibration amplitudes are better suppressed especially for values of feedback gain $b$ that are greater 0.4 under given parameters.

As increases $f$, the response in every period is independent; the lower branch will disappear gradually; thus, under certain values of feedback gain $b$, the response is one closed loop in every period.

4.3. Stable Feedback Parameter Combinations $(\tau, b)$. For a vibration system, the maximum displacement amplitude usually should be suppressed under a target level. Since the maximum displacement depends on the feedback parameters, how to choose the feedback gain and time delay will be explained by the following example.

Here, the given parameters $c=0.08, \Omega=1.2, k_{1}=-0.08$, $f=1.0$ are chosen and meanwhile the maximum amplitude limit $A$ is defined as 1.5. As shown in Figure 8, the three division loops are corresponding to the critical amplitude $A=1.5$ with the negative stiffness $k_{1}=-0.08,-0.5$, and 


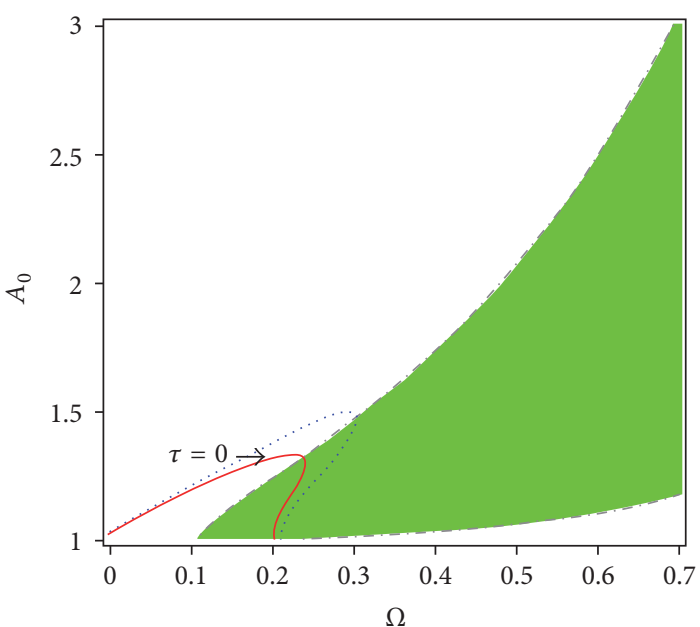

Uncontrolled

- Controlled

(a)

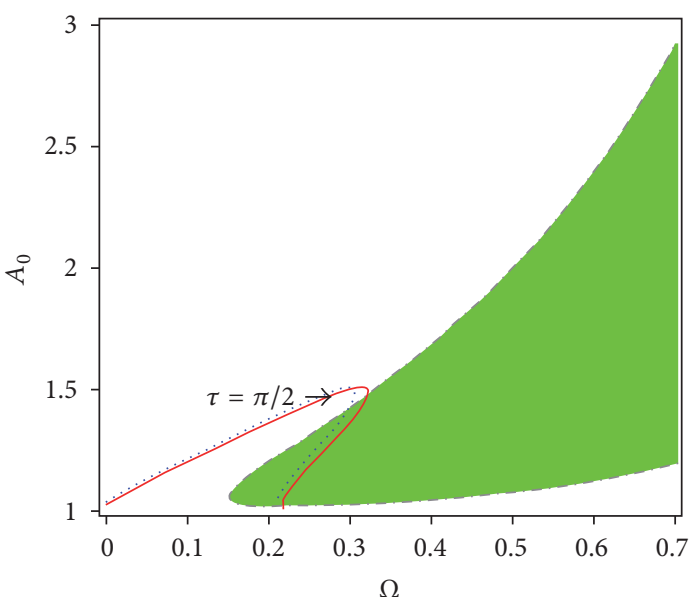

...... Uncontrolled

— Controlled

(c)

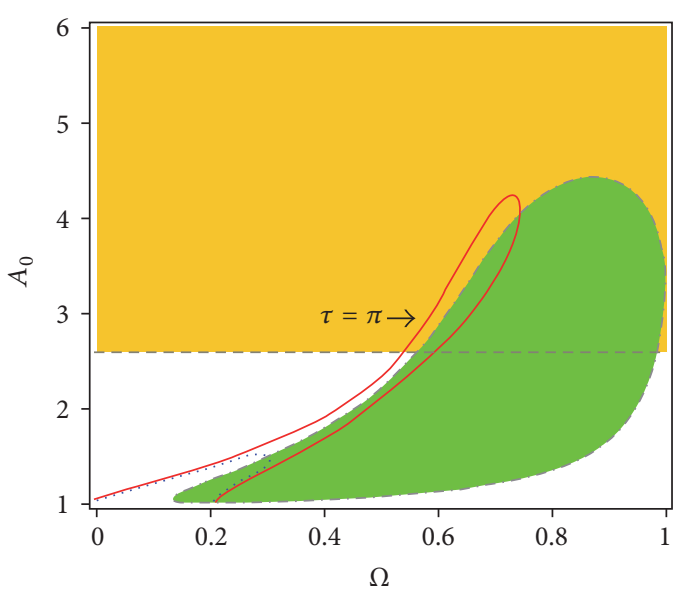

…. Uncontrolled

- Controlled

(e)

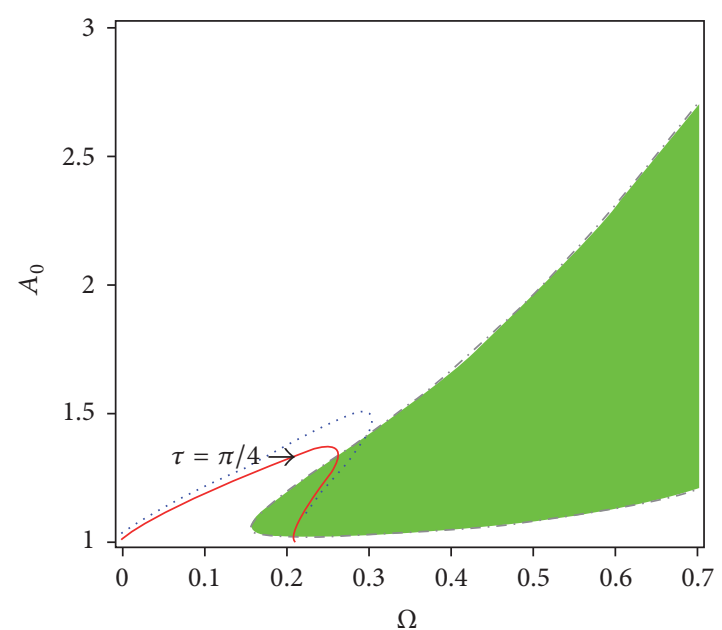

Uncontrolled

Controlled

(b)

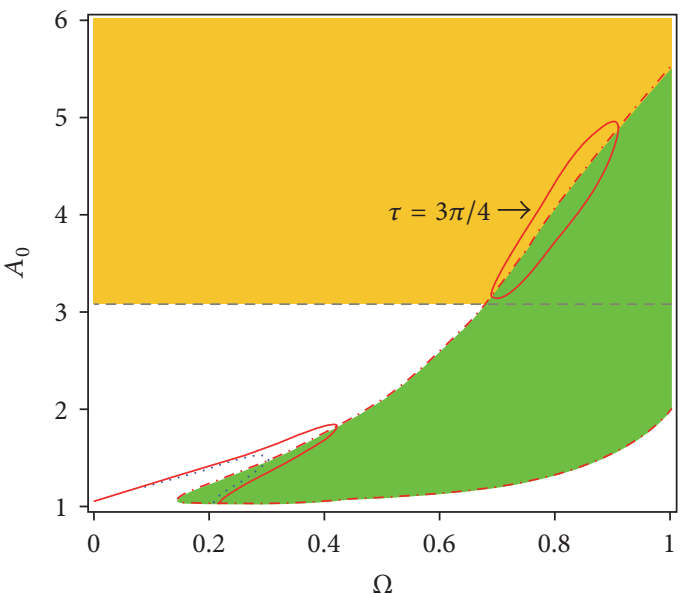

Uncontrolled

Controlled

(d)

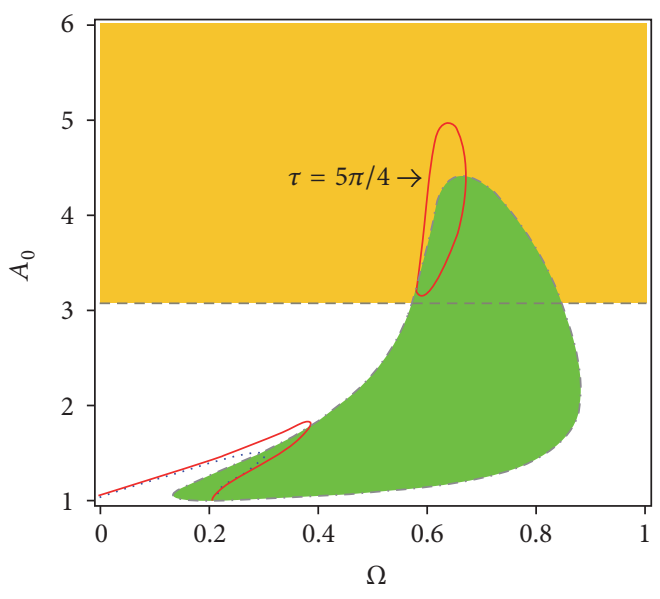

..... Uncontrolled

- Controlled

Figure 5: Continued. 


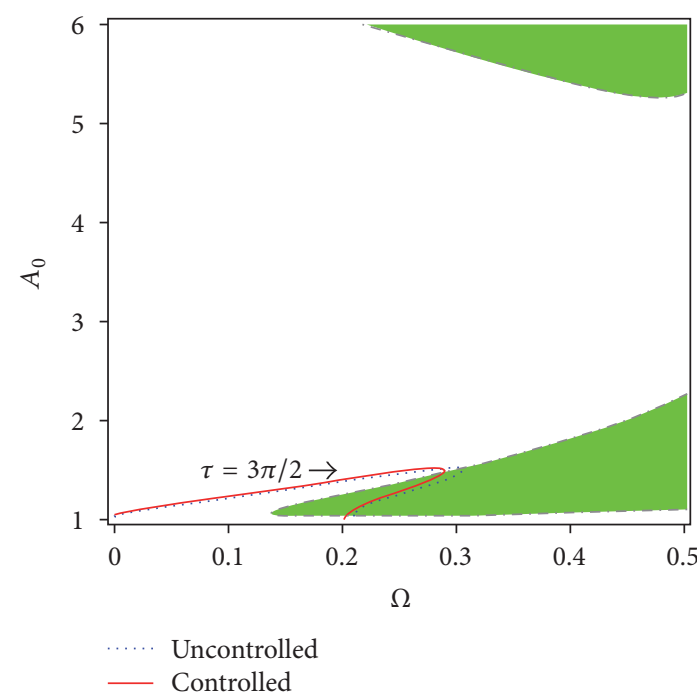

(g)

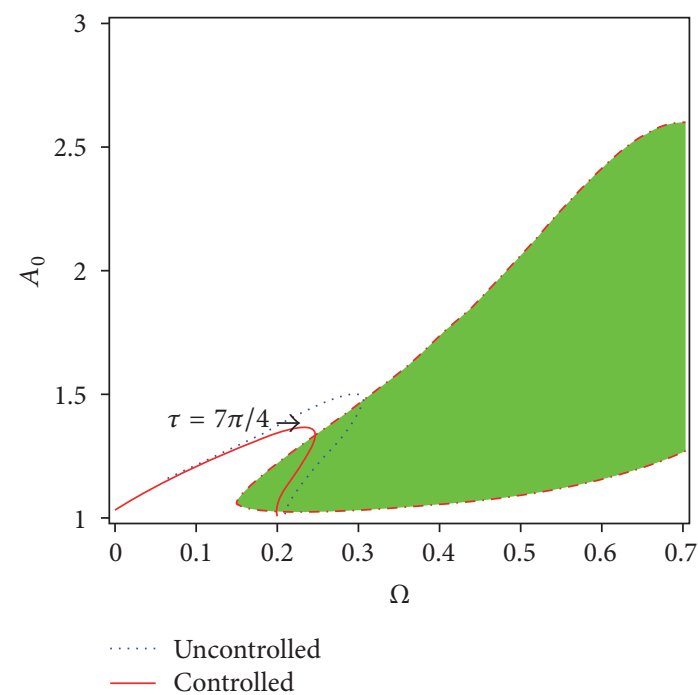

(h)

FIGURE 5: Comparison between the amplitude-frequency responses of controlled and uncontrolled systems: $c=0.2 ; b=0.02 ; f=0.3$. Solid lines: the responses of controlled system; dash lines: the responses of uncontrolled system; shaded regions: unstable regions. (a) $\tau=0.0$; (b) $\tau=\pi / 4$; (c) $\tau=\pi / 2$; (d) $\tau=3 \pi / 4$; (e) $\tau=\pi$; (f) $\tau=5 \pi / 4$; (g) $\tau=3 \pi / 2$; (h) $\tau=7 \pi / 4$.

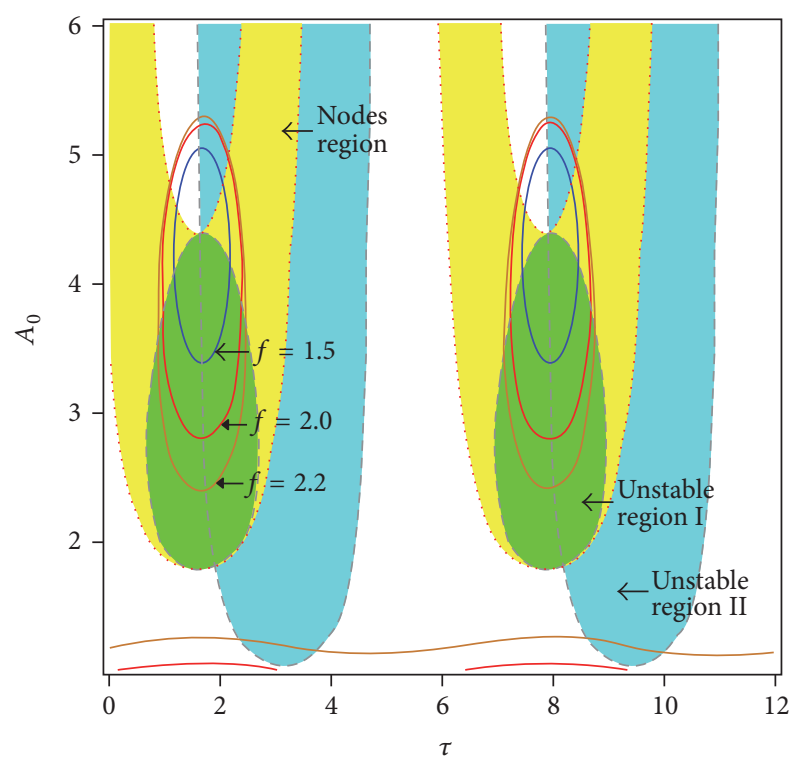

(a)

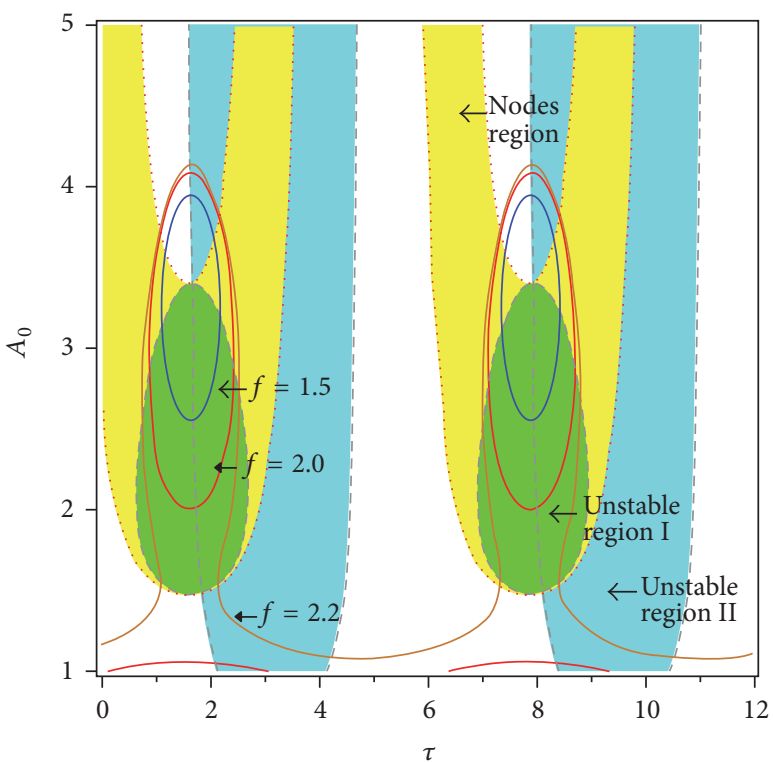

(b)

FIGURE 6: Effect of time delays on vibration amplitudes with different feedback gains. Solid lines: response curves (a) $b=0.05$; (b) $b=0.1$.

-0.8 , respectively. Outside each division loop, the amplitude governed by the parameter pairs $(\tau, b)$ is less than the specified limit value. However, not all parameter pairs located outside the division loop can satisfy the stability conditions. Hence, it is necessary to identify the corresponding stability boundaries which could exclude those feedback parameters falling in the unstable region. In Figure 8, the dash-dot, dot, and dash lines are the stability boundaries corresponding to the three different negative stiffness values $k_{1}=-0.08$, -0.5 , and -0.8 , respectively. Additionally, the shaded region surrounded by the black long-dash line is another part of the unstable regions, which is uniform in spite of different values of $k_{1}$.

Furthermore, the relation of the 3D figure of time delay, feedback gain, and negative stiffness is plotted for suppressing the maximum response amplitude $A=1.5$ denoted as cylinder-shaped surface $P$ in Figure 9. Correspondingly, the stability boundaries are also depicted as U-shape surface R and cylinder-shaped surface Q. When the parameter pairs are obtained outside the surface $\mathrm{P}$, the maximum amplitude 


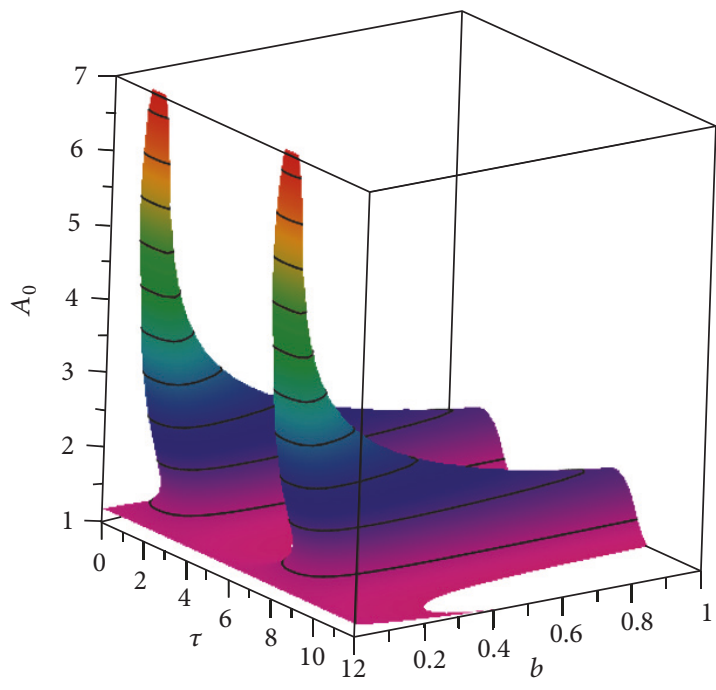

(a)

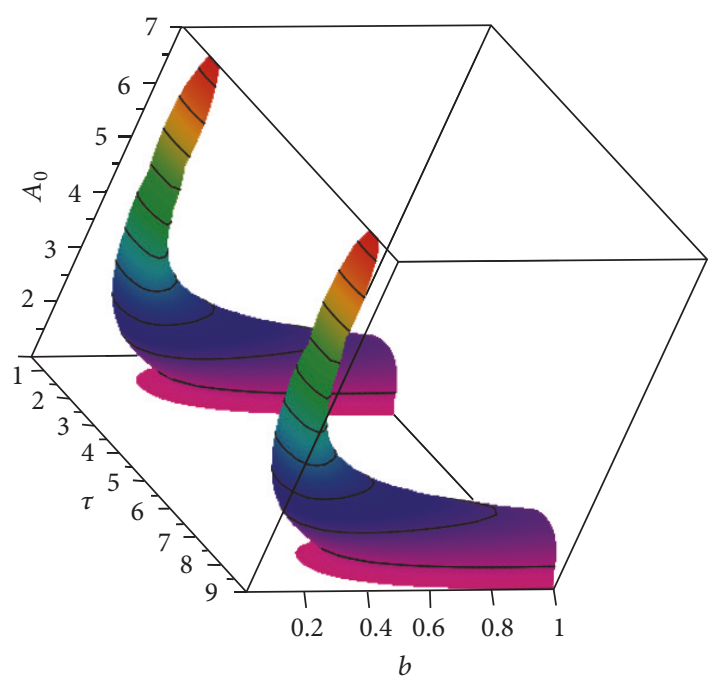

(c)

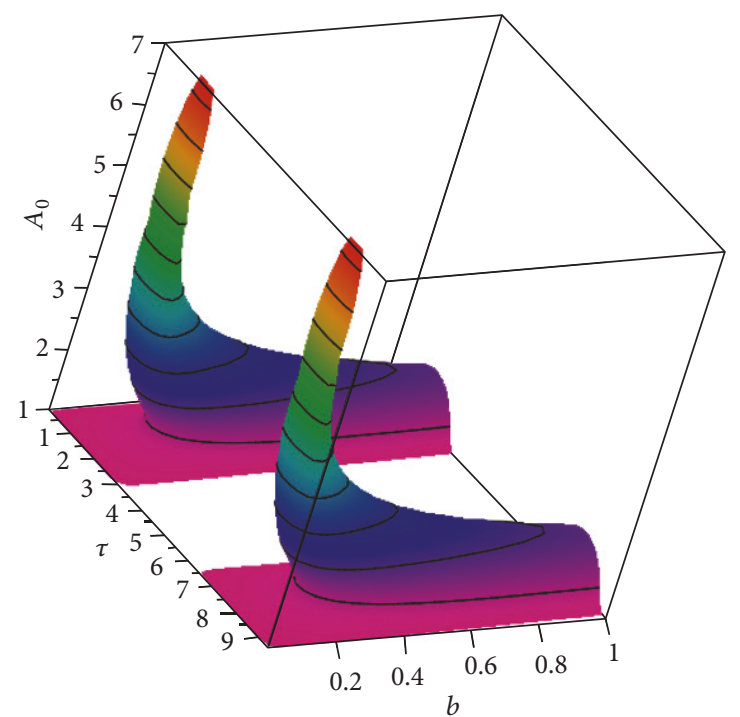

(b)

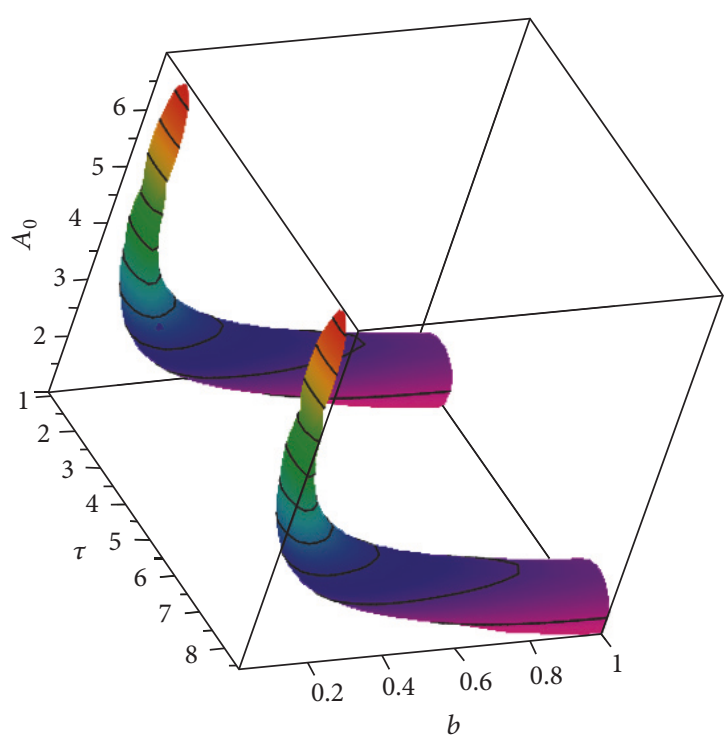

(d)

Figure 7: 3D surface plot of vibration amplitude of the system with changing feedback gains and time delays. (a) $f=2.2$; (b) $f=2.0$; (c) $f=1.8$; (d) $f=1.5$.

is smaller than 1.5. In order to meet the requirement of the stability conditions and suppress the maximum amplitude, simultaneously, the selection of the parameter pairs for $A<$ 1.5 should be exclude those points inside the surface $\mathrm{Q}$ and inside the U-shape surface R.

\section{Analysis of Mean-Square Responses}

Obviously, as shown in (25), the first-order steady-state moment $E A$ is equal to $A_{0}$; hence, only the second-order steady-state moment $E\left(A^{2}\right)$ is under consideration. For the simulation of narrow-band random noise, readers can refer to results obtained by Shinozuka and Jan [32] and by Zhu [33].

Figure 10 depicts the dependence of the mean square to excitation frequency under different excitation amplitude $f$ for $\widehat{\gamma}=0.01$ with time delays $\tau=\pi / 2$ and $\tau=3 \pi / 4$. Actually, under small noise intensity, the topological properties of system (2) (as shown in Figure 11) keep unchanged; therefore, the mean-square response characteristic is similar to that for the deterministic case.

To explain the effect of the noise intensity on the system response more clearly, the phase portraits and time histories are presented in Figure 11. The phase portraits confirm that under smaller noise intensity the topology property of the stochastic DPWS system keeps unchanged compared with the deterministic case, such as the case shown in Figure 11(b). Only the limit cycle becomes the diffused limit cycle which can be observed in Figure 11(b). However, under larger noise intensity, the phase trajectory has been chaotic as shown in Figures 11(c) and 11(f). 


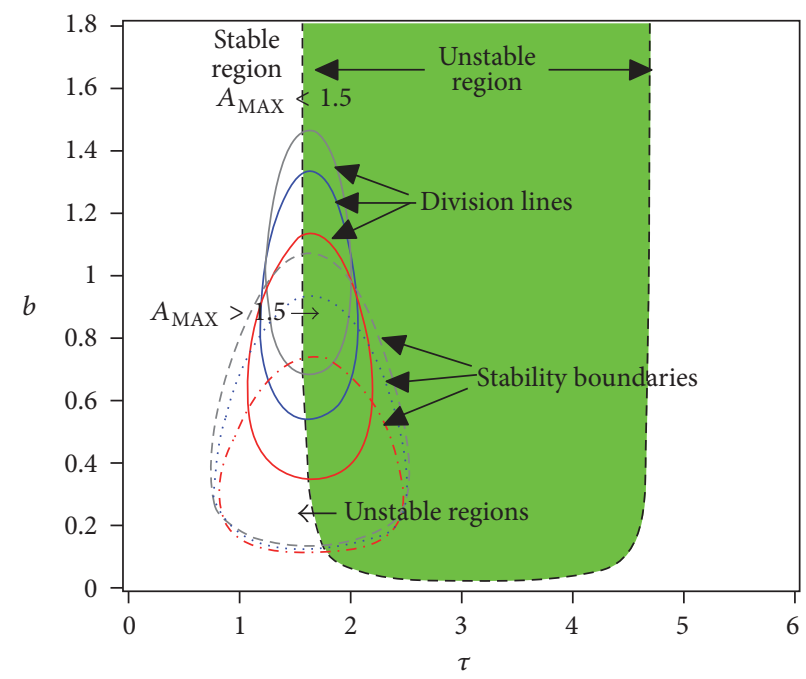

FIGURE 8: Design illustration of feedback gain and time delay.

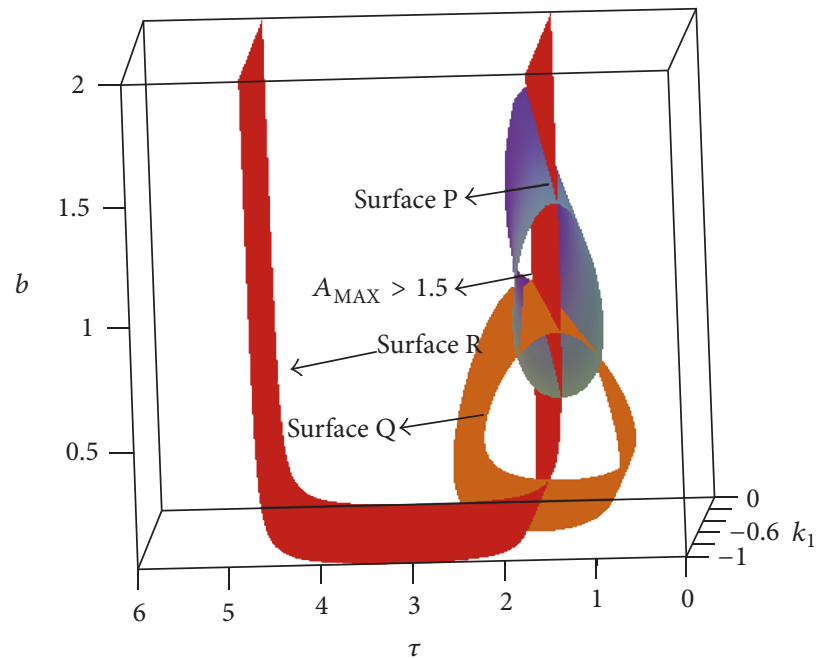

FIGURE 9: The relations of time delay, feedback gain, and negative stiffness for suppressing the given maximum displacement amplitude.

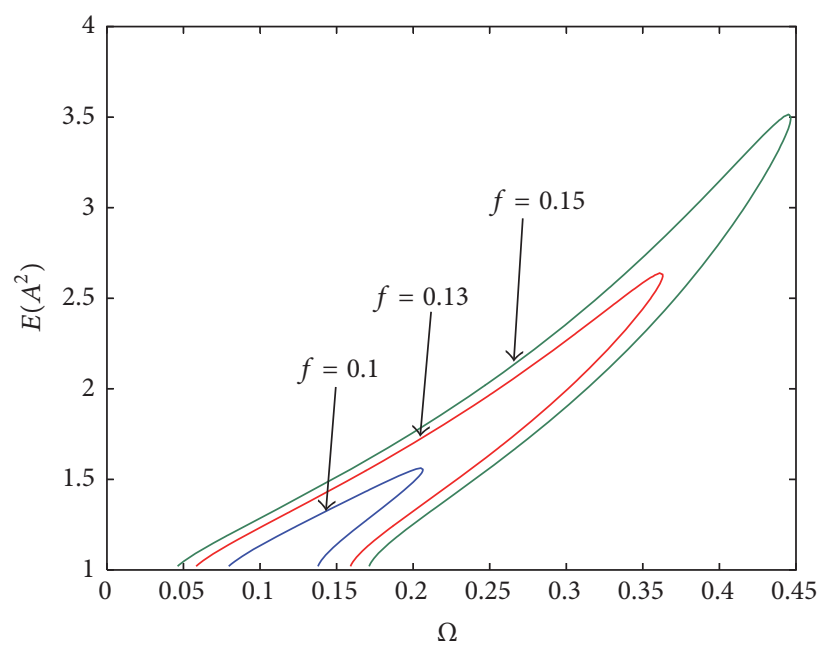

(a)

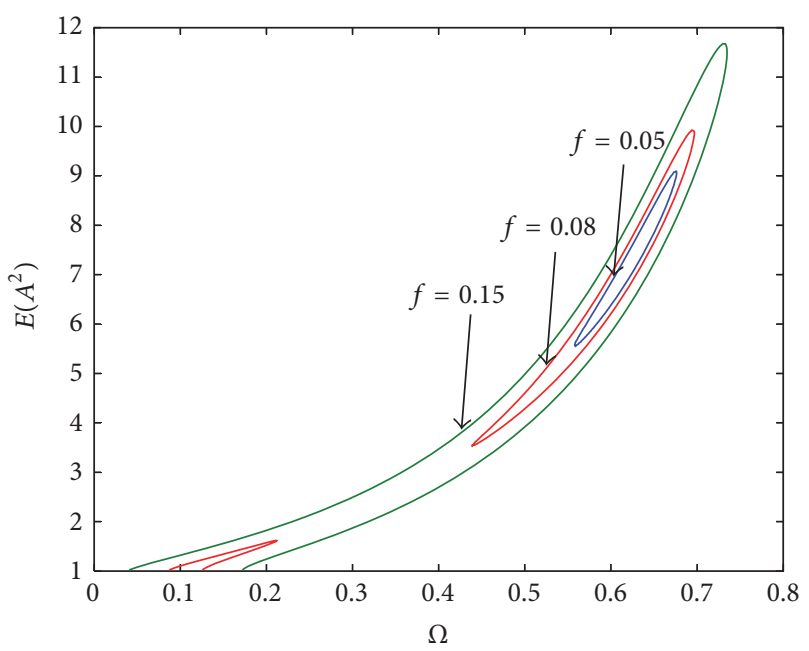

(b)

FIGURE 10: Mean-square responses under different external excitation amplitude $f$ for the noise intensity $\widehat{\gamma}=0.01$. (a) $\tau=\pi / 2$; (b) $\tau=3 \pi / 4$. 


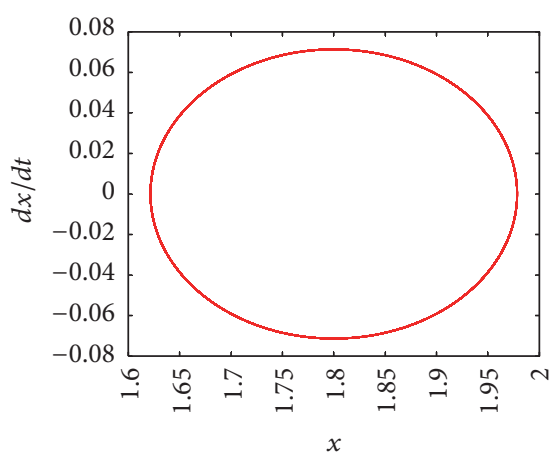

(a)

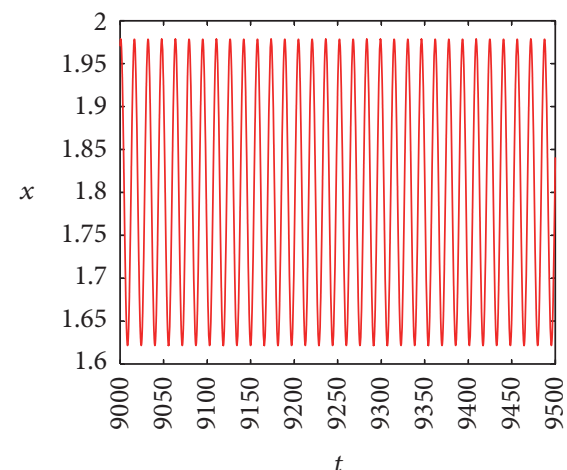

(d)

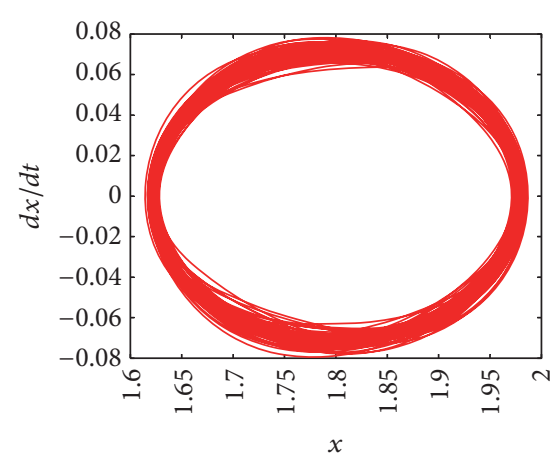

(b)

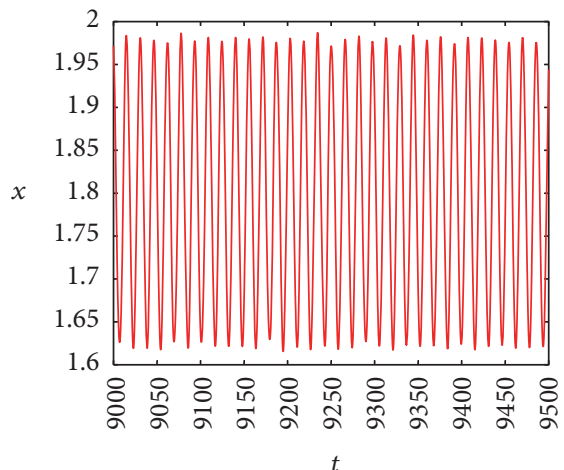

(e)

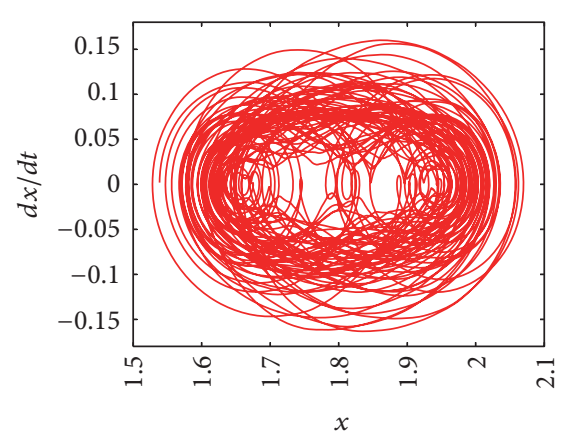

(c)

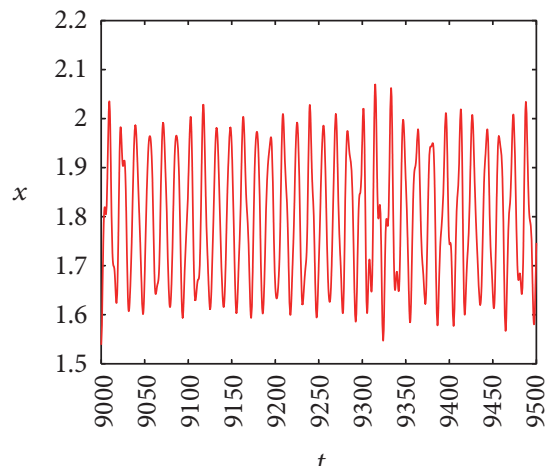

(f)

FIGURE 11: Phase portraits and time histories of the DPWS system under the parameters $\Omega=0.4 ; \tau=\pi / 2 ; f=0.15$. (a) Phase portraits for $\gamma=0.0$; (b) phase portraits for $\gamma=0.01$; (c) phase portraits for $\gamma=0.1$; (d) time histories for $\gamma=0.0$; (e) time histories $\gamma=0.01$; (f) time histories for $\gamma=0.1$.

\section{Conclusions}

In this paper, both the method of multiple scales and the moment method were adopted to study the nonlinear dynamical properties and the response performance of a DPWS system with negative stiffness under narrow-band random excitation. The frequency island phenomenon was found and verified under certain parameters. By means of equivalent damping, the performances of controlled system are compared to the system without control by some typical amplitude-frequency curves. Then, the dependence of the feedback parameters on the responses was analyzed, and meanwhile stability analysis of the closed-loop system was examined. It was found that, with the changes of the time delay, the response, the stability regions, and the distribution of the steady-state solutions appear periodically. And the critical relation of the time delay and feedback gain for suppressing the maximum response amplitude is a closed loop in every period rather than a continuous curve.

\section{Conflicts of Interest}

The authors declare that they have no conflicts of interest.

\section{Acknowledgments}

This work was supported by the National Natural Science Foundation of China (Grants nos. 11702201 and 11602184) and the Fundamental Research Funds for the Central Universities (Grant no. JBX170708).

\section{References}

[1] S. W. Shaw and P. J. Holmes, "A periodically forced piecewise linear oscillator," Journal of Sound and Vibration, vol. 90, no. 1, pp. 129-155, 1983.

[2] M. Wiercigroch and V. W. T. Sin, "Experimental study of a symmetrical piecewise base-excited oscillator," Journal of Applied Mechanics, Transactions ASME, vol. 65, no. 3, pp. 657-663, 1998.

[3] E. V. Karpenko, M. Wiercigroch, E. E. Pavlovskaia, and M. P. Cartmell, "Piecewise approximate analytical solutions for a Jeffcott rotor with a snubber ring," International Journal of Mechanical Sciences, vol. 44, no. 3, pp. 475-488, 2002.

[4] Q. Brandon, T. Ueta, D. Fournier-Prunaret, and T. Kousaka, "Numerical bifurcation analysis framework for autonomous piecewise-smooth dynamical systems," Chaos, Solitons and Fractals, vol. 42, no. 1, pp. 187-201, 2009.

[5] E. Pavlovskaia and M. Wiercigroch, "Low-dimensional maps for piecewise smooth oscillators," Journal of Sound and Vibration, vol. 305, no. 4-5, pp. 750-771, 2007.

[6] S. Banerjee and G. C. Verghese, Nonlinear Phenomena in Power Electronics, IEEE, 2001.

[7] M. Garcia, A. Chatterjee, A. Ruina, and M. Coleman, "The simplest walking model: Stability, complexity, and scaling," Journal of Biomechanical Engineering, vol. 120, no. 2, pp. 281-286, 1998. 
[8] J.-Y. Yoon and B. Kim, "Vibro-impact energy analysis of a geared system with piecewise-type nonlinearities using various parameter values," Energies, vol. 8, no. 8, pp. 8924-8944, 2015.

[9] J. Xu and J. Li, "Stochastic dynamic response and reliability assessment of controlled structures with fractional derivative model of viscoelastic dampers," Mechanical Systems and Signal Processing, vol. 72-73, pp. 865-896, 2016.

[10] A. Hassibi and S. Boyd, "Quadratic stabilization and control of piecewise-linear systems," in Proceedings of the American Control Conference (ACC '98), vol. 6, pp. 3659-3664, Philadelphia, $\mathrm{Pa}$, USA, June 1998.

[11] S. Pettersson and B. Lennartson, "LMI for stability and robustness of hybrid systems," in Proceedings of the 1997 American Control Conference. Part 3 (of 6), pp. 1714-1718, June 1997.

[12] O. Slupphaug and B. A. Foss, "Constrained quadratic stabilization of discrete-time uncertain non-linear multi-model systems using piecewise affine state-feedback," International Journal of Control, vol. 72, no. 7-8, pp. 686-701, 1999.

[13] X. Gao and Q. Chen, "Nonlinear analysis, design and vibration isolation for a bilinear system with time-delayed cubic velocity feedback," Journal of Sound and Vibration, vol. 333, no. 6, pp. 1562-1576, 2014.

[14] C. Cheng, S. Li, Y. Wang, and X. Jiang, "On the analysis of a high-static-low-dynamic stiffness vibration isolator with timedelayed cubic displacement feedback," Journal of Sound and Vibration, vol. 378, pp. 76-91, 2016.

[15] K. Zou and S. Nagarajaiah, "Study of a piecewise linear dynamic system with negative and positive stiffness," Communications in Nonlinear Science and Numerical Simulation, vol. 22, no. 1-3, pp. 1084-1101, 2015.

[16] D.-M. Huang, W. Xu, W.-X. Xie, and Q. Han, "Principal resonance response of a stochastic elastic impact oscillator under nonlinear delayed state feedback," Chinese Physics B, vol. 24, no. 4, Article ID 040502, 2015.

[17] H. Hu, E. H. Dowell, and L. N. Virgin, "Resonances of a harmonically forced Duffing oscillator with time delay state feedback," Nonlinear Dynamics, vol. 15, no. 4, pp. 311-327, 1998.

[18] C. Li, X. Liao, and R. Zhang, "Delay-dependent exponential stability analysis of bi-directional associative memory neural networks with time delay: An LMI approach," Chaos, Solitons and Fractals, vol. 24, no. 4, pp. 1119-1134, 2005.

[19] J. Zhou, T. Chen, and L. Xiang, "Adaptive synchronization of coupled chaotic delayed systems based on parameter identification and its applications," International Journal of Bifurcation and Chaos in Applied Sciences and Engineering, vol. 16, no. 10, pp. 2923-2933, 2006.

[20] J. Lü and G. Chen, "A time-varying complex dynamical network model and its controlled synchronization criteria," Institute of Electrical and Electronics Engineers. Transactions on Automatic Control, vol. 50, no. 6, pp. 841-846, 2005.

[21] C. S. Feng and R. Liu, "Response of Duffing system with delayed feedback control under bounded noise excitation," Archive of Applied Mechanics, pp. 1-9, 2012.

[22] D. Huang, W. Xu, W. Xie, and Y. Liu, "Dynamical properties of a forced vibration isolation system with real-power nonlinearities in restoring and damping forces," Nonlinear Dynamics, vol. 81, no. 1-2, pp. 641-658, 2015.

[23] N. A. Nayfeh and W. T. Baumann, "Nonlinear analysis of timedelay position feedback control of container cranes," Nonlinear Dynamics, vol. 53, no. 1-2, pp. 75-88, 2008.

[24] W. Molyneux, Supports for Vibration Isolation, HM Stationery Office, 1957.
[25] A. A. Sarlis, D. T. R. Pasala, M. C. Constantinou, A. M. Reinhorn, S. Nagarajaiah, and D. P. Taylor, "Negative stiffness device for seismic protection of structures," Journal of Structural Engineering (United States), vol. 139, no. 7, pp. 1124-1133, 2013.

[26] S. N. Madhekar and R. S. Jangid, "Use of pseudo-negative stiffness dampers for reducing the seismic response of bridges: a benchmark study," Bulletin of Earthquake Engineering, vol. 10, no. 5, pp. 1561-1583, 2012.

[27] D. T. R. Pasala, A. A. Sarlis, S. Nagarajaiah, A. M. Reinhorn, M. C. Constantinou, and D. Taylor, "Adaptive negative stiffness: New structural modification approach for seismic protection," Journal of Structural Engineering (United States), vol. 139, no. 7, pp. 1112-1123, 2013.

[28] D. T. R. Pasala, A. A. Sarlis, A. M. Reinhorn, S. Nagarajaiah, M. C. Constantinou, and D. Taylor, "Simulated bilinear-elastic behavior in a SDOF elastic structure using negative stiffness device: Experimental and analytical study," Journal of Structural Engineering (United States), vol. 140, no. 2, Article ID 04013049, 2014.

[29] R. Stratonovich, Topics in the Theory of Random Noise, vol. 2, Gordon and Breach, New York, NY, USA, 1967.

[30] A. H. Nayfeh and D. T. Mook, Nonlinear oscillations, John Wiley \& Sons, 2008.

[31] G. Schmidt and A. Tondl, Non-Linear Vibrations, Cambridge University Press, New York, NY, USA, 1986.

[32] M. Shinozuka and C. M. Jan, "Digital simulation of random processes and its applications," Journal of Sound and Vibration, vol. 25, no. 1, pp. 111-128, 1972.

[33] W. Q. Zhu, Random Vibration, Science Press, Beijing, China, 1998. 


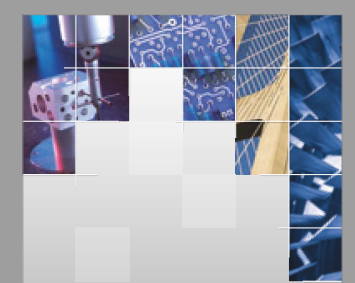

\section{Enfincering}
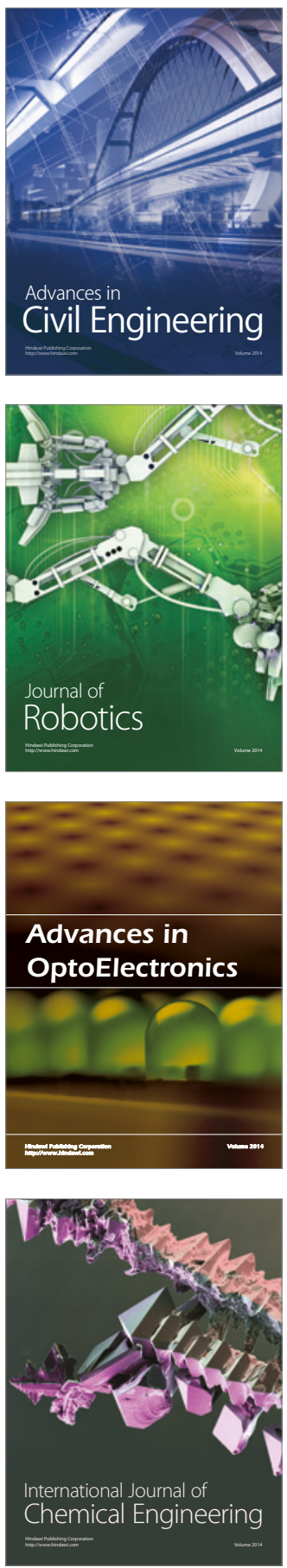

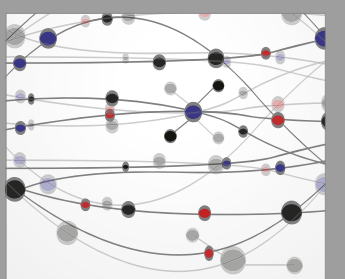

The Scientific World Journal

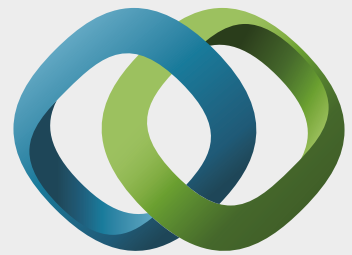

\section{Hindawi}

Submit your manuscripts at

https://www.hindawi.com
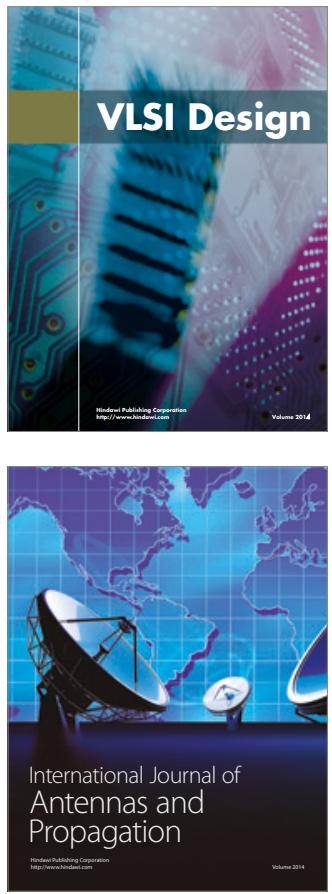

\section{Rotating}

Machinery
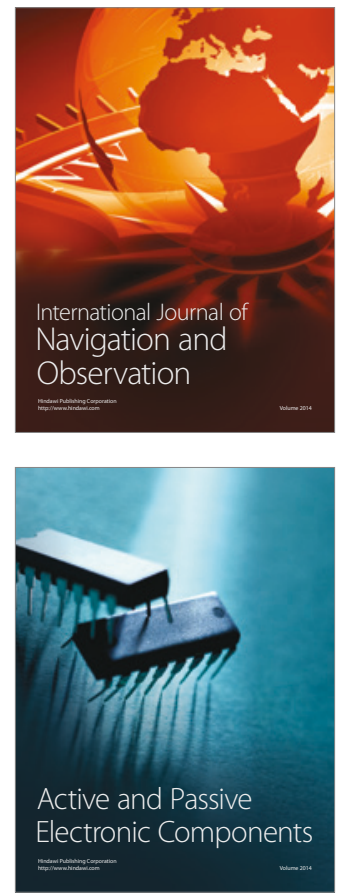
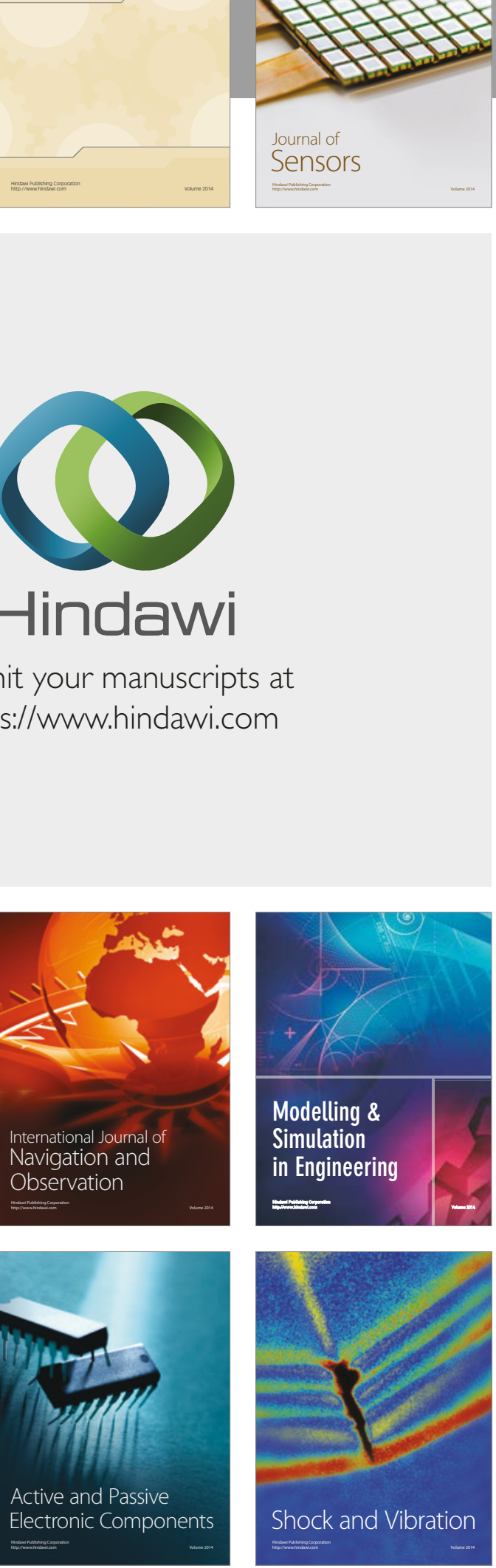
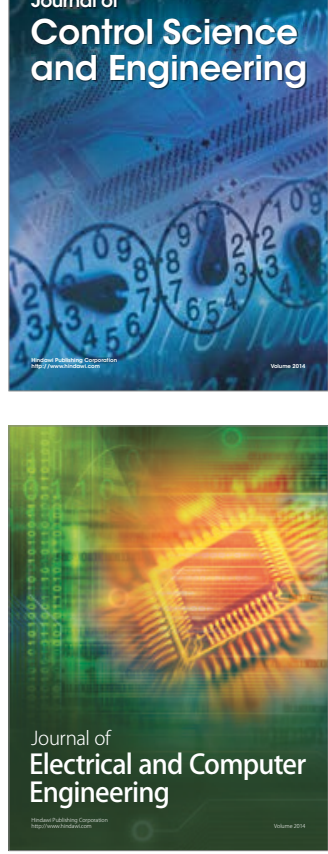

Distributed

Journal of

Control Science

and Engineering
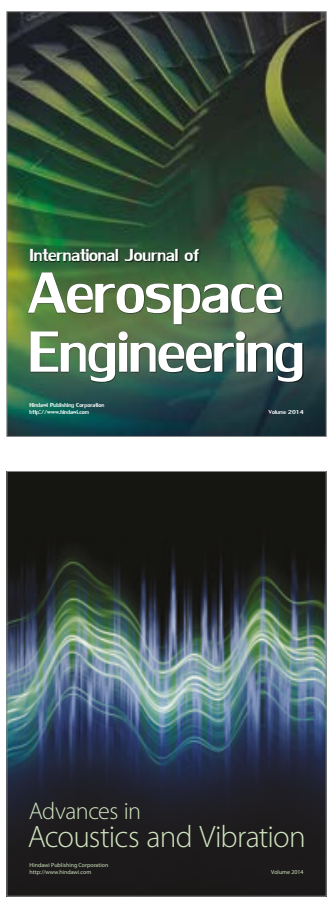

Sensor Networks 